\title{
Uncertainty analysis for the net-section-collapse failure criterion of circumferentially cracked cylinders for multiple arbitrary-shaped circumferential cracks
}

\author{
Rémi Dingreville ${ }^{1}$, Aubrey Eckert-Gallup, Cédric Sallaberry \\ Sandia National Laboratories, Albuquerque, NM 87185, USA
}

\begin{abstract}
In this manuscript, a generalized net-section-collapse (NSC) failure criterion of circumferentially cracked pipes with multiple arbitrary-shaped cracks is presented. This generalized NSC formulation is capable of predicting the NSC moment of a pipe with multiple arbitrary-shaped cracks distributed around the circumference of the pipe, regardless of whether they are distributed symmetrically or not. The case in which internal cracks straddle the compressive zone is accounted for in the present formulation. Closed form solutions are provided for the maximum moments of pipes containing multiple cracks with idealized shapes, namely constant depth, semi-elliptical and parabolic crack profiles. Through a series of examples, the results show the effectiveness and accuracy of the method. Using this method, quantification of the effect of the crack profile uncertainty on the maximum bending moment sustained by a cracked pipe is evaluated. It is demonstrated that while the uncertainty associated with the surface roughness of the crack profile has little to no effect on the maximum bending moment, irregular shape profiles have, not surprisingly, a large effect on the estimation of the maximum bending moment. In fact, it is mathematically shown that the uncertainty associated with the maximum bending moment
\end{abstract}

\footnotetext{
${ }^{1}$ Corresponding author. Tel.: 505-844-9083; E-mail: rdingre@sandia.gov
} 
is proportional to the magnitude of the crack profile uncertainty and inversely proportional to the square root of the uncertainty sampling size (i.e, surface roughness vs. irregular crack profile).

Keywords: Surface cracks, net section collapse, stability criterion, corrosion, ASME Section XI.

\section{Introduction}

Although the historical performance of piping systems in nuclear power plants, offshore drilling platforms, gas transmission lines and other applications has been exceptional, their increasing age has led to concerns regarding the unavoidable occurrence and growth of internal corrosion induced cracks (Office of Pipeline Safety, US Department of Transportation, 2004). As piping systems age, internal circumferential cracks and other degradation mechanisms can incur a reduction and loss of structural strength and an eventual failure (Kanninen et al., 1976; Cramlington, 1996). Clearly, regular inspections of piping systems and compliance with standards guidelines, such as the ASME Boiler and Pressure Vessel Code Section XI (1997) in the United States, the RSE-M Code (1997) in France, or the rules for fitness-for-service provided by the Japan Society of Mechanical Engineers (2008) in Japan, can reduce the risk of any undue failure caused by a lack of awareness regarding the integrity of the pipes.

Numerous models and computational analysis have been proposed for predicting the failure load of a pipe containing circumferential cracks. Among them, the net-sectioncollapse (NSC) analysis (Rahman and Wilkowski, 1998; Rahman, 1998; Lei and Budden, 2004; Kim et al., 2006; Staat and Vu, 2006; Li et al., 2010) is a simple and straightforward method for calculating the maximum load a cracked pipe can sustain assuming that failure is governed by the strength of the materials (generally based on an average between the yield stress and ultimate strength). Rahman and Wilkowski (1998) NSC analysis is 
however limited to a single symmetrical circumferential crack centered at the top of the pipe circumference. Recently, Li et al. (2010) extended the approach by Rahman and Wilkowski (1998) analysis to an analysis of a cracked pipe with multiple circumferential cracks with constant depths located above the neutral axis (i.e. all in tension). More detailed analyses (Han et al., 1999; Kim et al., 2002, 2003) are based on Finite Element Analysis (FEA) and explicitly model complex crack morphology and capture the threedimensional nature of the stress field. For example Han et al. (1999) computed the limit moment of local wall thinning pipe under pure bending by employing 3D elastic-plastic finite element analysis. Their results showed that the limit moment of pipe is affected not only by the width of a defect but also by the longitudinal length of a defect. For a given longitudinal length of a defect overpassing some critical value, the authors showed that results from the NSC criterion are in good agreement with the results from finite element analysis.

The above mentioned analyses, both analytical and computational, are limited to idealized crack profiles (either constant depth, semi-elliptical depth, or parabolic depth) and generally apply to a single circumferential crack. However actual cracks present in piping systems have variable profiles around the pipe circumference (Rahman and Wilkowski, 1998). Choosing an idealized crack constitutes a conservative approach. Furthermore, API579 (Anderson and Osage, 2000), BS7910 (British Standards Institution, 2000) and other codes have defects interaction rules that can require multiple defects to be characterized by a circumscribing ellipse for example reinforcing the conservatism for estimating plastic collapse. Few studies in the literature have looked at the impact of the variability and stochastic nature of cracks on the reduction and loss of structural strength of piping systems. Most notably, Rahman (1997) described the development of a probabilistic fracture mechanics model for analyzing circumferential through-walled cracked pipes subjected to bending loads. First- and second-order reliability methods (FORM/SORM) as 
well as importance sampling and Monte Carlo Simulations were used for conducting a structural reliability analysis and for determining the characteristics of the J-integral for a circumferential through wall cracked pipe as a function of the applied bending moment.

The present work proposes a generalized method for NSC analysis of cracked pipes with multiple arbitrary crack shapes. This method can be used to calculate the maximum load of a circumferentially cracked pipe containing multiple non-interacting internal surface cracks with variable depth subjected to combined bending and tension (pressure induced) loads. Note that since the present formulation is a NSC analysis, the hoop stress is not considered. The effect of hoop stresses on net-section-collapse load for single circumferential cracked pipes has been considered for example in Oh et al. (2008). This manuscript will focus on answering one key question: Can we quantify how the uncertainty on the NSC moment is affected by the uncertainty on the cracks profiles? Examples illustrating the idealized cases for a pipe containing multiple cracks with constant depth profiles, semi-elliptical profiles and parabolic profiles will be used as a starting point for discussion on the impact of the uncertainty of the crack profile on the NSC analysis.

The manuscript is organized as follows. Section 2 introduces the generalized netsection-collapse method for a pipe containing multiple surface cracks with arbitrary distribution and discusses the closed-form solutions for constant depth, semi-elliptical and parabolic cracks. Section 3 discusses the treatment and characterization of crack profile uncertainty. Section 4 provides a discussion on the main findings of the work while section 5 provides recommendations for future investigations.

\section{The generalized net-section-collapse method for a pipe containing multiple surface cracks with arbitrary distribution}

Let us consider a pipe containing multiple independent and dissimilar internal circumferential surface cracks with variable depth profiles as shown in Fig. 1. The pipe has a 
mean radius, $R_{m}$, an average wall thickness $t$ (consequently the internal radius $R_{i}$ is simply defined as $R_{i}=R_{m}-t / 2$ ) and is subjected to an internal pressure $p$ and an externally applied bending moment $M$ about the $x$-axis. Let $N$ be the number of independent dissimilar internal surface cracks. Each surface crack " $j$ " is characterized by (i) its angular position $\gamma_{j}$ (center of the crack) measured from the $y$-axis, (ii) its arbitrary crack depth profile $a_{j}(\xi)$ as a function of an angular coordinate measured from the $y$-axis, and (iii) its total angular length $2 \theta_{j}$. For now, the following analysis assumes that the cracks are distributed symmetrically about the bending plane ( $y$-axis). The asymmetric case will be treated later in this manuscript. Having symmetry about the $y$-axis ensures a symmetrical distribution of the stresses. In the asymmetric case, the internal bending moment may have components about both the $x$ - and $y$-axes, violating the equilibrium condition. As previously noted by Rahman and Wilkowski (1998), this does not mean that a real pipe cannot have an asymmetric distribution of circumferential cracks subjected to an external bending moment, rather the NSC criterion may not be the most appropriate method.

Fig. 2 shows the internal stress distribution in a cracked pipe wall containing $N$ dissimilar cracks. The stress distribution has to be in equilibrium with the applied load, i.e. the internal pressure $p$ and the bending moment $M$. The hoop stress from the pressure and its resulting multi-axial state of stress were not considered. In Fig. 2, the neutral axis $\beta_{N}$ corresponds to the location of stress inversion.

\subsection{Case for which all the cracks are in tension}

This case corresponds to a configuration of cracks for which $\gamma_{j}+\theta_{j}<\pi-\beta_{N}$ and $\gamma_{j}-\theta_{j}>\beta_{N}-\pi$ for all cracks “ $j$ ”, $\forall j \in[1, N]$. The equilibrium of the axial forces yields,

$$
\sigma_{f} R_{m} t\left[\sum_{j=1}^{N} \int_{\gamma_{j}-\theta_{j}}^{\gamma_{j}+\theta_{j}}-\frac{a_{j}(\xi)}{t} d \xi\right]+2 \sigma_{f} R_{m} t\left(\pi-\beta_{N}\right)-2 \sigma_{f} R_{m} t \beta_{N}=p \pi R_{i}^{2},
$$


where $\sigma_{f}$ is the flow stress. Equation (1) can be used to deduce the neutral axis $\beta_{N}$,

$$
\beta_{N}=\frac{1}{2}\left[\pi-\frac{p \pi R_{i}^{2}}{2 \sigma_{f} R_{m} t}-\frac{1}{2} \sum_{j=1}^{N} \int_{\gamma_{j}-\theta_{j}}^{\gamma_{j}+\theta_{j}} \frac{a_{j}(\xi)}{t} d \xi\right] .
$$

From the moment equilibrium, the net section collapse moment $M$ is given by,

$$
\begin{aligned}
M & =2 \sigma_{f} R_{m}^{2} t\left[\int_{0}^{\beta_{N}} \cos \xi d \xi+\int_{0}^{\pi-\beta_{N}} \cos \xi d \xi-\frac{1}{2} \sum_{j=1}^{N} \int_{\gamma_{j}-\theta_{j}}^{\gamma_{j}+\theta_{j}} \frac{a_{j}(\xi)}{t} \cos \xi d \xi\right] \\
& =2 \sigma_{f} R_{m}^{2} t\left[2 \sin \beta_{N}-\frac{1}{2} \sum_{j=1}^{N} \int_{\gamma_{j}-\theta_{j}}^{\gamma_{j}+\theta_{j}} \frac{a_{j}(\xi)}{t} \cos \xi d \xi\right],
\end{aligned}
$$

for which the neutral axis $\beta_{N}$ is given by equation (2). Note that both equations (2) and (3) involve integral formulations of crack profiles $a_{j}(\xi)$ requiring in most cases a numerical evaluation of these expressions.

It can be easily shown that equations (2) and (3) are consistent with those of Rahman and Wilkowski (1998) for a cracked pipe containing a single symmetrical crack $(N=1$ and $\left.\gamma_{j}=0\right)$ and those of Li et al. (2010) for multiple cracks with a constant depth profile symmetrically distributed about the circumference.

\subsection{Case for which some cracks are in compression or straddle the compressive zone}

When some cracks are completely in compression or straddle the compressive zone, equations (2)-(3) need to be adjusted to account for crack closure and to allow the transmission of any compressive forces. As shown in Fig. 3, among the $N$ cracks present in the circumference of the pipe, several configurations need to be differentiated:

- $N_{1}\left(0 \leq N_{1} \leq N\right)$ cracks " $j$ " for which if $\gamma_{j}+\theta_{j}<\pi-\beta_{N}$ and $\gamma_{j}-\theta_{j}>\beta_{N}-\pi$, the cracks are completely in tension (see Fig. 3 (a)).

- $N_{2}\left(0 \leq N_{2} \leq 1\right)$ cracks on the right of the centerline for which if $\beta_{N}-\pi<\gamma_{j}+\theta_{j} \leq$ $\pi-\beta_{N}$ and $-\pi-\beta_{N} \leq \gamma_{j}-\theta_{j}<\beta_{N}-\pi$, the cracks are partially in compression (see Fig. 3 (b)). 
- $N_{3}\left(0 \leq N_{3} \leq 1\right)$ cracks on the left of the centerline for which if $\pi-\beta_{N}<\gamma_{j}+\theta_{j} \leq$ $\pi+\beta_{N}$ and $\pi-\beta_{N}>\gamma_{j}-\theta_{j} \geq \beta_{N}-\pi$, the cracks are partially in compression (see Fig. 3 (c)).

- $N_{4}\left(0 \leq N_{4} \leq 1\right)$ cracks for which if $\gamma_{j}+\theta_{j}>\pi-\beta_{N}$ and $\gamma_{j}-\theta_{j}<\beta_{N}-\pi$, both crack tips are straddling the compressive zone from above the neutral axis (see Fig. 3 (d)).

- $N_{5}\left(0 \leq N_{5} \leq 1\right)$ cracks for which if $\gamma_{j}>0$ then $\gamma_{j}+\theta_{j}>\pi+\beta_{N}$ and $\gamma_{j}-\theta_{j}<\pi-\beta_{N}$, and if $\gamma_{j}<0$ then $\gamma_{j}+\theta_{j}>\beta_{N}-\pi$ and $\gamma_{j}-\theta_{j}<-\pi-\beta_{N}$, the cracks straddle the tensile zone from below the neutral axis (see Fig. 3 (e)).

- $N_{6}$ cracks $\left(N_{6}=N-\sum_{j=1}^{5} N_{i}\right)$ are completely in compression (see Fig. 3 (f)).

From the force equilibrium, the calculation of the neutral axis becomes a self-consistent formulation in which the compressive zone implicitly self adjusts such that,

$$
\begin{aligned}
4 \beta_{N} & =2 \pi-\frac{p \pi R_{i}^{2}}{\sigma_{f} R_{m} t}-\sum_{j=1}^{N_{1}} \int_{\gamma_{j}-\theta_{j}}^{\gamma_{j}+\theta_{j}} \frac{a_{j}(\xi)}{t} d \xi-\sum_{j=1}^{N_{2}} \int_{\beta_{N}-\pi}^{\gamma_{j}+\theta_{j}} \frac{a_{j}(\xi)}{t} d \xi-\sum_{j=1}^{N_{3}} \int_{\gamma_{j}-\theta_{j}}^{\pi-\beta_{N}} \frac{a_{j}(\xi)}{t} d \xi \\
& -\sum_{j=1}^{N_{4}} \int_{\beta_{N}-\pi}^{\pi-\beta_{N}} \frac{a_{j}(\xi)}{t} d \xi-\sum_{j=1}^{N_{5}}\left[\int_{\gamma_{j}-\theta_{j}}^{\operatorname{sgn}\left(\gamma_{j}\right) \pi-\beta_{N}} \frac{a_{j}(\xi)}{t} d \xi+\int_{\operatorname{sgn}\left(\gamma_{j}\right) \pi+\beta_{N}}^{\gamma_{j}+\theta_{j}} \frac{a_{j}(\xi)}{t} d \xi\right]
\end{aligned}
$$

Due to physical consideration, note that equation (4) has a converged solution. This can easily be proven by transforming the integral formulation into a series. The convergence is proven by recurrence.

From the moment equilibrium, the net section collapse moment $\mathrm{M}$ is given by,

$$
M=2 \sigma_{f} R_{m}^{2} t\left[2 \sin \beta_{N}-\frac{1}{2} \sum_{j=1}^{N_{r}} \int_{\gamma_{j}^{\prime}-\theta_{j}^{\prime}}^{\gamma_{j}^{\prime}+\theta_{j}^{\prime}} \frac{a_{j}(\xi)}{t} \cos \xi d \xi\right],
$$

where $\beta_{N}$ is given by (4), and $N_{r}=N_{1}+N_{2}+N_{3}+N_{4}+2 N_{5}$ (note that cracks totally in compression are omitted from the summation due to crack closure) and the adjusted crack position $\gamma_{j}^{\prime}$ and crack half-length $\theta_{j}^{\prime}$ are defined such that: 
- If all the cracks are in tension, $\gamma_{j}^{\prime}=\gamma_{j}$ and $\theta_{j}^{\prime}=\theta_{j}$.

- If a crack is straddling the compressive zone on the right side of the centerline, $\gamma_{j}^{\prime}=\beta_{N}+\theta_{j}^{\prime}-\pi$ and $\theta_{j}^{\prime}=\frac{1}{2}\left[\gamma_{j}+\theta_{j}+\pi-\beta_{N}\right]$.

- If a crack is straddling the compressive zone on the left side of the centerline, $\gamma_{j}^{\prime}=$ $\pi-\beta_{N}-\theta_{j}^{\prime}$ and $\theta_{j}^{\prime}=\frac{1}{2}\left[\pi-\beta_{N}-\gamma_{j}+\theta_{j}\right]$.

- If both crack tips are straddling the compressive zone from above the neutral axis, $\gamma_{j}^{\prime}=0$ and $\theta_{j}^{\prime}=\pi-\beta_{N}$.

- If both crack tips are straddling the tensile zone from below the neutral axis, the crack needs to be split into two cracks such that $\gamma_{j, 1}^{\prime}=\pi-\beta_{N}-\theta_{j, 1}^{\prime}$ and $\theta_{j, 1}^{\prime}=$ $\frac{1}{2}\left[\operatorname{sgn}\left(\gamma_{j}\right) \pi-\beta_{N}-\gamma_{j}+\theta_{j}\right]$; and $\gamma_{j, 2}^{\prime}=\beta_{N}+\theta_{j, 2}^{\prime}-\pi$ and $\theta_{j, 2}^{\prime}=\frac{1}{2}\left[\gamma_{j}+\theta_{j}-\operatorname{sgn}\left(\gamma_{j}\right) \pi-\beta_{N}\right]$.

Equations (1)-(5) are valid for circumferential surface cracks with any arbitrary shape provided that they are symmetrically distributed about the pipe circumference with respect to the pipe centerline. In the event that surface cracks are not symmetrically distributed, as was mentioned in Li et al. (2010), the position of the coordinate axes have to be established in order to minimize the bending moment to obtain a conservative estimation.

\subsection{Asymmetric distribution of cracks around the pipe circumference}

If the distribution of surface cracks around the circumference of the pipe is asymmetric with respect to its centerline, new positions of the coordinate axis have to be defined in order to minimize the failure bending moment for conservative estimation. As shown in Fig. 4 , if we denote by $\varphi$ the rotation angle that minimizes the bending moment, the net section collapse moment after rotation $M^{\prime}$ is simply given by,

$$
M^{\prime}=2 \sigma_{f} R_{m}^{2} t\left[2 \sin \beta_{N}-\frac{1}{2} \sum_{j=1}^{N} \int_{\gamma_{j}-\theta_{j}-\varphi}^{\gamma_{j}+\theta_{j}-\varphi} \frac{a_{j}(\xi)}{t} \cos \xi d \xi\right] .
$$


The positions of the coordinate axes for which the net section collapse moment is at a minimum satisfy the following condition,

$$
M^{\prime}=\min M, \forall \varphi \in[-\pi, \pi] .
$$

This rotated configuration can be assimilated as the weighed equivalent symmetric case. Note that, in some cases for which all the cracks are idealized and in tension, and assuming that $M^{\prime}$ is a smooth function (i.e. at least $C^{1}$ ), the solution to equation (7) can be reduced to,

$$
\frac{\partial M^{\prime}}{\partial \varphi}=0 .
$$

For both of these conditions ( $C^{1}$ function and all cracks in tension), equation (6) reduces to only one harmonic and, as such, this equation will lead to the global minimum or maximum for $\varphi \in[-\pi, \pi]$. We will show in section 4.1 that the condition in equation (8) may not necessarily be a satisfactory condition for finding the minimum bending moment.

\subsection{Discretization of crack profiles}

In the most general case, equations (4)-(5) do not possess a closed-form solution, rather a discretization of the crack profiles is necessary. The crack profiles are discretized around the circumference of the pipe with a constant angular resolution of $\Delta \vartheta$ independent of the crack length $\theta_{j}$ and regardless of the crack " $j$ ". As such the number of angular discretized coordinates for crack " $j$ " is simply given by,

$$
\eta_{j}=\left\lceil\frac{2 \theta_{j} R_{i}}{\Delta \vartheta}\right\rceil,
$$

where $\lceil\cdot\rceil$ denotes the ceiling function (i.e. smallest integer greater than the actual value). Note that in this case, the size of discretized steps may vary slightly from crack to crack, but this approach allows exactly the entire length of each crack to be covered with roughly the same angular discretized resolution. As illustrated in Fig. 5, the integration of a given 
crack profile $a_{j}(\xi)$ over some range around the circumference of the pipe can be evaluated using discrete values of $a_{j}(\xi)$ by numerical quadrature such that,

$$
\int a_{j}(\xi) d \xi=\sum_{k=1}^{\eta_{j}} a_{j}\left(\xi_{k}\right) \frac{2 \theta_{j} R_{i}}{\eta_{j}},
$$

where $\xi_{k}$ is the $k^{\text {th }}$ discrete value of the angular coordinate $\xi, a\left(\xi_{k}\right)$ is the crack depth at angular location $\xi_{k}$.

Similarly to the generalized NSC formulation, some logical requirements for cracks in tension and compression need to be considered based on the previously defined cases for crack location:

- Trivially, for all cracks that are entirely in tension, the steps that are in tension are $k$ such that $1 \leq k \leq \eta_{j}$.

- For cracks that are in compression on the left with respect to the polar coordinates, we must define a left bound, $L B_{1}$, the step defining the approximate boundary between the compressive and tensile zones. $L B_{1}$ satisfies the following relationship:

$$
L B_{1}=\left\lfloor\eta_{j} \frac{\beta_{N}-\pi-\gamma_{j}+\theta_{j}+\varphi}{2 \theta_{j}}\right\rfloor,
$$

where $\lfloor\cdot\rfloor$ denotes the floor function (i.e. the largest integer smaller than the actual value). Thus the discrete steps $k$ are in tension for all $k$ such that $L B_{1} \leq k \leq \eta_{j}$.

- For cracks that are in compression on the right with respect to the polar coordinates, we must define a right bound, $R B_{1}$, the step defining the approximate boundary between the compressive and tensile zones. $R B_{1}$ satisfies the following relationship:

$$
R B_{1}=\left\lceil\eta_{j} \frac{\pi-\beta_{N}-\gamma_{j}+\theta_{j}+\varphi}{2 \theta_{j}}\right\rceil
$$

Thus the discrete steps $k$ are in tension for all $k$ such that $1 \leq k<R B_{1}$. 
- For cracks for which both crack tips are straddling the compressive zone above the neutral axis, we can use the same right bound, $R B_{1}$, and left bound, $L B_{1}$, defined above. Then discrete steps $k$ are in tension for all $k$ such that $L B_{1} \leq k<R B_{1}$.

- For cracks that straddle the tensile zone from below the neutral axis, we have two sub-cases, $\gamma_{j}>0$ and $\gamma_{j}<0$ :

- For $\gamma_{j}>0$ we must define a new bound on the left with respect to the polar coordinates, $L B_{2}$. $L B_{2}$ satisfies the following relationship:

$$
L B_{2}=\left\lfloor\eta_{j} \frac{\beta_{N}+\pi-\gamma_{j}+\theta_{j}+\varphi}{2 \theta_{j}}\right\rfloor .
$$

If $R B_{1}$ remains defined as above, then the discrete steps $k$ are in tension for all $k$ such that $1 \leq k<R B_{1}$ and $L B_{2} \leq k \leq \eta_{j}$.

- For $\gamma_{j}<0$, we must define a new bound on the right with respect to the polar coordinates, $R B_{2} . R B_{2}$ satisfies the following relationship:

$$
R B_{2}=\left\lceil\eta_{j} \frac{-\pi-\beta_{N}-\gamma_{j}+\theta_{j}+\varphi}{2 \theta_{j}}\right\rceil .
$$

If $L B_{1}$ remains defined as above, then the discrete steps $k$ are in tension for all $k$ such that $1 \leq k<R B_{2}$ and $L B_{1} \leq k \leq \eta_{j}$.

Using the discretization scheme described above, we can now numerically estimate the position of the neutral axis such that,

$$
4 \beta_{N}=2 \pi-\frac{p \pi R_{i}^{2}}{\sigma_{f} R_{m} t}-\sum_{j=1}^{N} \sum_{k=1}^{\eta_{j}} \frac{D\left[a_{j}\left(\xi_{k}\right)\right]}{t} \frac{2 \theta_{j}}{\eta_{j}} .
$$

where

$$
D\left[a_{j}\left(\xi_{k}\right)\right]= \begin{cases}a_{j}\left(\xi_{k}\right) & \text { if } \xi_{k} \text { is in the tensile zone. } \\ 0 & \text { if } \xi_{k} \text { is in the compressive zone. }\end{cases}
$$


In order to find the bending moment following this discretization, we must first define the center location for each discrete step, $\gamma_{j, k}$ such that,

$$
\gamma_{j, k}=\gamma_{j}-\theta_{j}+\left(k-\frac{1}{2}\right) \frac{2 \theta_{j}}{\eta_{j}} .
$$

The numerical estimate of the bending moment is subsequently given by,

$$
M=2 \sigma_{f} R_{m}^{2} t\left[2 \sin \beta_{N}-\sum_{j=1}^{N} \sum_{k=1}^{\eta_{j}} \frac{D\left[a_{j}\left(\xi_{k}\right)\right]}{t} \frac{\theta_{j}}{\eta_{j}} \cos \left(\gamma_{j, k}-\varphi\right)\right]
$$

In the case of the asymmetric distribution of cracks around the circumference of the pipe, we can simply vary the value of $\varphi$ in a discretized manner between $-\pi$ and $\pi$, and subsequently estimate the value of $\beta_{N}$ and the bending moment $M$. Based on our previously stated condition in equation (7) for the position of the coordinate axes for which the net section collapse is at a minimum, we retain the solution of $\varphi$ that leads to the minimum value of the bending moment over the discrete variation of $\varphi$.

\subsection{Special cases}

As shown in Fig. 6, three distinct idealized crack geometries were considered: constantdepth crack, elliptical crack, and parabolic crack. The mathematical formulation for each profile is as follows,

$$
a_{j}(\xi)= \begin{cases}a_{0, j} & \text { for constant-depth cracks. } \\ a_{0, j} \sqrt{1-\left(\frac{\xi-\gamma_{j}}{\theta_{j}}\right)^{2}} & \text { for semi-elliptical cracks. } \\ a_{0, j}\left[1-\left(\frac{\xi-\gamma_{j}}{\theta_{j}}\right)^{2}\right] & \text { for parabolic cracks. }\end{cases}
$$

Note the difference in formulation for parabolic cracks between the present formulation and that of Rahman (1998). A typo in the formulation by Rahman (1998) leads $a_{j}(\xi)$ to reach its maximum value at $\xi=-\theta_{j}$ and its minimum at $\theta_{j}$, rather than being centered 
around $\gamma_{j}$. The present formulation corrects this minor misrepresentation of the crack profile, resulting in slightly different results for the parabolic case (in the case of one crack) compared to those of Rahman (1998) but more in line with the results obtained for the two other crack profiles. For these three cases, analytical solutions to equations (2)-(5) can be easily obtained.

\subsubsection{Constant-depth cracks}

For a cracked pipe containing constant-depth cracks, the crack depth profile $a_{j}(\xi)$ is simply represented by $a_{j}(\xi)=a_{0, j}$, where $a_{0, j}$ is the depth of crack " $j$ " at the center of the crack. The generalized NSC equations (2)-(3) reduce in this case to simple closed-form expressions:

$$
\beta_{N}=\frac{1}{2}\left[\pi-\frac{p \pi R_{i}^{2}}{2 \sigma_{f} R_{m} t}-\sum_{j=1}^{N} \frac{a_{0, j} \theta_{j}}{t}\right]
$$

for the neutral axis and,

$$
M=2 \sigma_{f} R_{m}^{2} t\left[2 \sin \beta_{N}-\sum_{j=1}^{N} \frac{a_{0, j}}{t} \cos \gamma_{j} \sin \theta_{j}\right],
$$

for the net-section-collapse moment.

\subsubsection{Parabolic cracks}

For a cracked pipe containing cracks with a parabolic crack depth profile, the crack depth profile $a_{j}(\xi)$ is simply represented by $a_{j}(\xi)=a_{0, j}\left[1-\left(\left(\xi-\gamma_{j}\right) / \theta_{j}\right)^{2}\right]$, where $a_{0, j}$ is the nominal depth of crack " $j$ ". The generalized NSC equations (2)-(3) reduce in this case to closed-form expressions,

$$
\beta_{N}=\frac{\pi}{2}-\frac{p \pi R_{i}^{2}}{4 \sigma_{f} R_{m} t}-\sum_{j=1}^{N} \frac{a_{0, j} \theta_{j}}{3 t},
$$

for the neutral axis and,

$$
M=4 \sigma_{f} R_{m}^{2} t\left[\sin \beta_{N}-\sum_{j=1}^{N} \frac{2 a_{0, j} \cos \gamma_{j}\left(\sin \theta_{j}-\theta_{j} \cos \theta_{j}\right)}{t \theta_{j}^{2}}\right],
$$


for the net-section collapse moment.

\subsubsection{Semi-elliptical cracks}

For a cracked pipe containing cracks with a semi-elliptical crack depth profile, the crack depth profile $a_{j}(\xi)$ is simply represented by $a_{j}(\xi)=a_{0, j}\left[1-\left(\left(\xi-\gamma_{j}\right) / \theta_{j}\right)^{2}\right]^{1 / 2}$, where $a_{0, j}$ is the nominal depth of crack " $j$ ”. The generalized NSC equations (2)-(3) reduce in this case to closed-form expressions,

$$
\beta_{N}=\frac{\pi}{2}-\frac{p \pi R_{i}^{2}}{4 \sigma_{f} R_{m} t}-\frac{1}{8} \sum_{j=1}^{N} \frac{a_{0, j} \pi \theta_{j}}{t},
$$

for the neutral axis and,

$$
\begin{aligned}
M & =\sigma_{f} R_{m}^{2} t\left[4 \sin \beta_{N}\right. \\
& \left.-\frac{a_{0, j}}{t} \sum_{m=1}^{\infty} \theta_{j}^{2 m+1} \frac{\Gamma\left(\frac{2 m+1}{2}\right) \Gamma\left(\frac{3}{2}\right)}{\Gamma(m+2)} \sum_{l=m}^{\infty} \frac{(-1)^{l}}{(2 m) !(2 l-2 m) !} \gamma_{j}^{2 l-2 m}\right],
\end{aligned}
$$

for the net-section collapse moment, with the function $\Gamma(m)$ defined by,

$$
\Gamma(m)=\int_{0}^{\infty} x^{m} \exp (-x) d x, \text { for } x>0 .
$$

\section{Treatment and characterization of crack profiles uncertainty}

As illustrated in the above, cracks are usually represented with an idealized shape, the most common profiles being constant depth, parabolic or semi-elliptical. In reality, due to material heterogeneities and the inherent stochastic character of surface cracking and corrosion processes, the shape of cracks can substantially deviate from these idealized conceptions. Furthermore, crack growth mechanisms such as fatigue do not generally lead to the often assumed idealized defect shapes. These deviations may manifest themselves at different length scales with respect to the crack length: (i) at the smaller scale in the form 
of surface roughness or (ii) at larger scale in the form of irregular crack profiles. Fig. 7 illustrates these deviations over the idealized cases. These variations can be represented as a correction toward the idealized shape that is characterized with an uncertainty distribution. The corrective term accounting for the crack profile uncertainty can be considered either as a sum (reflecting how much the true shape deviates from the reference) or a product (representing the ratio of deviation from the reference). In this section we describe the treatment of the uncertainty and its characterization in the context of the Net Section Collapse method for multiple circumferential surface cracks by characterizing the uncertainty on the crack profiles either as an additive contribution or a multiplicative contribution over the nominal crack profile and demonstrate the equivalence that can be made between the two.

\subsection{Crack profiles uncertainty as an additive variation over the nominal crack profile}

First let us look at the uncertainty of the crack profile as an additive correction over the idealized crack profile. In this case, the crack depth $a_{j}(\xi)$ for any given crack " $j$ " is represented as the sum of the idealized crack depth $a_{0, j}(\xi)$ and a deviation term $\epsilon_{j}(\xi)$ such that

$$
a_{j}(\xi)=a_{0, j}(\xi)+\epsilon_{j}(\xi),
$$

where $\epsilon_{j}(\xi)$ is a random variable whose distribution reflects the type and strength of the uncertainty. Note that $\epsilon_{j}(\xi)$ is sampled from a distribution whose expected value $E\left(\epsilon_{j}\right)$ is 0 .

The uncertainty of the crack profile from the idealized crack can be described by its magnitude and its frequency. In terms of $\epsilon_{j}(\xi)$, the magnitude can be represented by the standard deviation $\sigma\left(\epsilon_{j}\right)$, while the frequency can be represented by the sample size $n_{s, j}$ for crack " $j$ " (i.e. how many times the value deviates from the idealized crack profile). In other words, for a high sampling size and a low standard deviation, one would consider the 
uncertainty of the crack profile to be due to surface roughness, while for a small sampling size and a high standard deviation, one would consider the uncertainty of the crack profile to be due to irregular profiles over the idealized profile. Note that the sampling size can vary from crack to crack, however for convenience and clarity in the rest of this manuscript, we assume a constant sampling size (i.e. $n_{s, j}=n_{s}, \forall j \in[1, N]$ ).

In this case, the change $\Delta F$ in the force equilibrium equation (1) can simply be expressed by,

$$
\Delta F=-\frac{1}{2 t} \sum_{j=1}^{N} \int_{\gamma_{j}-\theta_{j}}^{\gamma_{j}+\theta_{j}} \epsilon_{j}(\xi) d \xi \text { with } E\left(\epsilon_{j}\right)=0 .
$$

Given the fact that an integral formulation is used to estimate the effect of $\epsilon_{j}$ on $\Delta F$, one can sort each sampled value of $\epsilon_{j}$ and represent their variation as a Cumulative Distribution Function $(\mathrm{CDF})$. The mean of the uncertainty is zero, causing the effect of the uncertainty on the force equilibrium to decrease when the standard deviation tends to zero or when the sample size increases. As a consequence, it is now clear that, based on the aforementioned remark, the effect of the surface roughness (for a given amplitude) is minimal. Going back to the analysis of the force equilibrium, note that the location of the crack is irrelevant in equation (28), such that the integration can be carried over the interval $\left[0,2 \theta_{j}\right]$ and,

$$
\int_{\gamma_{j}-\theta_{j}}^{\gamma_{j}+\theta_{j}} \epsilon_{j}(\xi) d \xi=\int_{0}^{2 \theta_{j}} \epsilon_{j}(\xi) d \xi=\int_{0}^{2 \theta_{j}} \sum_{m=1}^{n_{s}} I_{m}\left[\epsilon_{j, m}(\xi)\right] d \xi
$$

with,

$$
I_{m}\left[\epsilon_{j, m}(\xi)\right]= \begin{cases}\epsilon_{j, m} & \text { if } \xi \in\left[\frac{2(m-1)}{n_{s}} \theta_{j}, \frac{2 m}{n_{s}} \theta_{j}\right] \\ 0 & \text { otherwise }\end{cases}
$$

After some calculation, the integration of equation (29) reduces to,

$$
\int_{\gamma_{j}-\theta_{j}}^{\gamma_{j}+\theta_{j}} \epsilon_{j}(\xi) d \xi=\frac{2 \theta_{j}}{n_{s}} \sum_{m=1}^{n_{s}} \epsilon_{j, m}
$$


Clearly from equation (31), by discretizing the randomness in the surface crack profile and sampling it, the change in force equilibrium $\Delta F$ can simply be defined by the uncertainty in the average estimate of the distribution representing the deviation from an idealized crack using a sample of size $n_{s}$. The standard error of the mean is estimated as the standard deviation of the population divided by the square root of the sample size. As a result, the change $\Delta F$ in force equilibrium resulting from the uncertainty on the crack profile can be estimated as being equal to,

$$
\sum_{j=1}^{N} \int_{\gamma_{j}-\theta_{j}}^{\gamma_{j}+\theta_{j}} \epsilon_{j}(\xi) d \xi \simeq \pm \sum_{j=1}^{N} 2 \theta_{j} \frac{\sigma\left(\epsilon_{j}\right)}{\sqrt{n_{s}}} .
$$

If the uncertainty in $\epsilon_{j}$ is represented using a normal distribution (i.e. $\epsilon_{j} \sim \mathcal{N}\left(0, \sigma\left(\epsilon_{j}\right)\right)$ then a t-distribution with $n_{s}-1$ degrees of freedom can be used to represent the uncertainty in the force equilibrium equation. The result is what would be expected intuitively: the force equilibrium will be more affected by the uncertainty when the amplitude of the uncertainty is increasing and/or the frequency is reduced (i.e. irregular crack profile). One interesting consequence of equation (32) lies in the fact that one can expect the force equilibrium to be affected linearly by the standard deviation (i.e. amplitude) and to be inversely proportional to the square root of the sampling size (i.e. period).

The change $\Delta M$ in the moment equilibrium equation (3) is twofold. The first of the changes mentioned above affect $\beta_{N}$ in a similar fashion (with variation coming from the change in force equilibrium).The second change affects the terms in the cracked region of the circumference of the pipe. Concerning the second of these changes, it can be expressed as,

$$
\Delta M_{1}=-\sigma_{f} R_{m}^{2} t \sum_{j=1}^{N} \int_{\gamma_{j}-\theta_{j}}^{\gamma_{j}+\theta_{j}} \epsilon_{j}(\xi) \cos \xi d \xi .
$$

A similar discretization used for the force equilibrium term can be applied to decompose 
this set of integrals, leading to the following estimation,

$$
\begin{array}{r}
\Delta M_{1}=-\sigma_{f} R_{m}^{2} t \sum_{j=1}^{N} \sum_{m=1}^{n_{s}} \epsilon_{j, k}[- \\
\sin \left(\gamma_{j}+\frac{2(m-1)-n_{s}}{n_{s}} \theta_{j}\right) \\
\left.+\sin \left(\gamma_{j}+\frac{2 m-n_{s}}{n_{s}} \theta_{j}\right)\right] .
\end{array}
$$

A first-order Taylor series expansion of the sine function can be used to obtain an approximation of this sum such that,

$$
\Delta M_{1} \simeq \mp \sigma_{f} R_{m}^{2} t \sum_{j=1}^{N} 2 \theta_{j} \frac{\sigma\left(\epsilon_{j}\right)}{\sqrt{n_{s}}} .
$$

Once again the result is as expected intuitively with the moment equilibrium being more affected by the uncertainty when the amplitude of the uncertainty is growing and/or the frequency is reduced.

\subsection{Crack profiles uncertainty as an multiplicative variation over the nominal crack pro-} file

When the uncertainty is applied as a multiplicative correction over the nominal idealized crack profile, the crack depth $a_{j}(\xi)$ for any given crack " $j$ " can be represented as the product of the idealized crack depth $a_{0, j}(\xi)$ and a deviation term $\epsilon_{j}(\xi)$ such that

$$
a_{j}(\xi)=a_{0, j}(\xi) \epsilon_{j}(\xi)
$$

where again $\epsilon_{j}(\xi)$ is a random variable whose distribution reflects the type and strength of the uncertainty. Note that $\epsilon_{j}(\xi)$ in this case now has to be sampled from a distribution that needs to be strictly positive and with an expected value of 1 . When using a multiplier, the equations leading to a change in force equilibrium and moment equilibrium are more complex and simplify only when the idealized crack shape is constant. As such, for the case of constant depth, the results presented for uncertainty as a multiplier are then 
equivalent to the results presented for uncertainty as a sum. For other variation of crack shape, the equations lead to an integral of two convoluted functions (one representing the crack shape and the other the uncertainty), whose solution would be specific to the distribution selected and the crack shape considered. No simple derivations can be found and a numerical approach is preferred.

Algorithmically, the use of discretization to estimate the equations simplifies the introduction of uncertainty in the cracks. Whether the sum or multiplication is selected, the uncertainty is sampled according to its frequency (sample size) and amplitude (range of the distribution) and applied along the crack to the crack depth. The equations of force equilibrium and moment equilibrium can be estimated with the newly defined shape.

\section{Results and Discussion}

In this section several numerical examples based the formulation developed above are presented to show the appropriateness and effectiveness of the proposed NSC estimation for pipes containing multiple circumferential cracks of arbitrary shapes.

\subsection{Analytical solutions for idealized cracks}

We first consider a pipe with mean radius $R_{m}=254 \mathrm{~mm}$, and a wall thickness of $t=$ $25.4 \mathrm{~mm}$, while the material flow stress is assumed to be $\sigma_{f}=300 \mathrm{MPa}$. The pipe is subjected to a combined bending moment and constant internal pressure of $p=15.51 \mathrm{MPa}$. Comparison between the various crack profiles is shown in Fig. 8. The variation of the normalized net section collapse moment $M / M_{0}$ is displayed as a function of the normalized crack depth $\theta / \pi$ for various ratios of the normalized maximum depth $a / t$. The normalizing moment $M_{0}$ is simply obtained from classical mechanics of materials and equation (3) when no cracks are present. As expected, the moment-carrying capacity of the pipe decreases with increasing crack coverage (both in terms of angle and crack depth). It is 
interesting to note the similarity and consistency in behavior between the various crack profiles. This was not the case in Rahman (1998) which contained a typo in the equation describing the parabolic crack profile (Rahman's equation for the parabolic crack profile reads $a(\xi)=(1-\xi / \theta)^{2}$ but should have read $\left.a(\xi)=1-(\xi / \theta)^{2}\right)$.

With that in mind, we now compare the present method to reported experimental data to gauge the accuracy of its predictions for various crack profiles. Fig. 9 compares the experimental results from Hasegawa et al. (2007) to the generalized net section collapse formulation presented in this manuscript for a pipe specimen containing two circumferential cracks symmetrically distributed around its circumference with respect to the $y$-axis. Hasegawa et al. (2007) conducted four-point bending experiments without any pressure on a 4-in diameter pipe containing two cracks fabricated by electric discharge machining. For these experiments, the reported outer diameter of the pipe is $114.8 \mathrm{~mm}$ with a wall thickness $t$ of $8.6 \mathrm{~mm}$. The pipe is made out of 304 stainless steel with a measured flaw stress of $\sigma_{f}=491 \mathrm{MPa}$. The measured depth of the surface cracks is reported to be $a / t=0.74$ with the crack angle of $\theta=\pi / 6$. Three data points were obtained by varying the angular distance $\alpha$ between the cracks, with $\alpha \in[0, \pi / 6]$. From the results shown in Fig. 9, we find a good agreement between the results obtained from our generalized net section collapse methodology and the experimental results. While the formulation assuming cracks with a constant depth profile underestimates the experimental data, the formulations assuming that the crack profile is either parabolic or semi-elliptical overestimate the experimental results. Clearly, with such formulation, the crack profiles could be optimized and calibrated to perfectly match experimental data points. Such a task is beyond the scope of the present manuscript.

As mentioned above, the proposed methodology to estimate the moment-carrying capacity of a pipe containing multiple cracks with arbitrary profiles can be used as an appropriate and effective rupture estimation method. In the rest of the manuscript, unless 
mentioned otherwise, we consider a pipe with the materials and geometry parameters used to generate Fig. 8. The example illustrated in Fig. 10 and Fig. 11 clarifies the effect of the number of cracks on the evolution of the net section collapse moment as a function of the combined circumferential crack coverage for both constant crack depth profile (see Fig. 11(a)) and semi-elliptical crack profile (see Fig. 11(b)). Cracks are distributed evenly around the circumference of the pipe. Note that for the cases for which we have two and four circumferential cracks, for small crack coverage, one crack may be in complete compression. A noticeable difference between the four cases can be clearly observed between the single crack configuration and the multiple crack configuration. The main explanation for this difference is due to the fact that in the configuration for which multiple cracks are present, more pipe ligaments in the tensile zone of the pipe carry the load than in the case of a single crack with an equivalent crack coverage. Additionally, it should be noted that, in the case of constant crack depth, when the combined crack coverage reaches the total circumference of the pipe (i.e. $\theta / \pi=1$ ), the estimation of the limit load carrying capacity of the pipe converges towards the same value regardless of the number of cracks. As expected, this is due to the fact that for that limit, all of the cases (in terms of the number of cracks) are equivalent to having a single crack along the circumference of the pipe. On the contrary, in the case of semi-elliptical or parabolic crack profiles, the above mentioned note is no longer valid since that, for a given combined crack coverage $\theta / \pi$, the uncracked cross section of the pipe will differ based on the number of cracks distributed around its circumference.

We now turn our attention to relevance and significance of the formulation in the case of an asymmetric distribution of cracks around the pipe circumference. Fig. 12(a) illustrates the variation of the normalized net section collapse moment with respect to the rotation angle $\varphi$ in the cases of a pipe containing two, three and four circumferential cracks, respectively. We notice that, as we rotate the positions of the coordinate axes in order 
to minimize the failure bending moment for conservative estimation, an $\mathrm{N}$-fold rotational symmetry is observed for $N$ cracks with a variation of $M / M_{0}$ of about $7 \%$. It is interesting to note that the variation decreases as the number of cracks increases. While it seems that in Fig. 12(a) the variation of $M / M_{0}$ is perfectly smooth, the variation is not a perfect harmonic. If we look at the contribution to the bending moment from each individual crack in Fig. 12(b), we can see how the compressive zone impacts the perfect harmonic. This effect is accentuated by the number of cracks and their area coverage. As such, this explains the decreases in amplitude of the variation of $M / M_{0}$ with respect to the rotation angle $\varphi$.

Fig. 13(a) and Fig. 13(b) emphasize the possible existence of local extrema as we rotate the positions of the coordinate axes to minimize the failure bending moment. These local extrema do not necessarily correspond to the global minimum used for the conservative estimation. Fig. 13(a) clearly shows that when no rotation is applied, the distribution of cracks actually corresponds to a local extrema (i.e. $\partial M^{\prime} / \partial \varphi=0$ ). A negative rotation would lead to a local minimum, but a positive rotation gives the true global minimum. Similarly, Fig. 13(b) illustrates similar observations. It is noteworthy to observe that in this case if we were to use equation (8) as a condition to find the minimum, the opposite effect would be found. Both of the examples discussed above serve as a justification for using equation (7) as a necessary and satisfactory condition for finding the minimum failure bending moment as opposed to using equation (8) as it was proposed in $\mathrm{Li}$ et al. (2010) for example. .

\subsection{Effect of crack profile uncertainty on the NSC moment}

As mentioned in section 3, we have have considered the uncertainty associated with crack profiles as a corrective term either as an additive or as a multiplicative variation over the nominal crack profiles. The uncertainty considered in the examples below follow a uniform distribution but other distributions could be used as well. Fig. 14 presents an il- 
lustration of the effects of the uncertainty associated with both the sampling size $n_{s}$ (see Fig. 14(a)), and the standard deviation $\sigma\left(\epsilon_{j}\right)$ (see Fig. 14(b)) in the case of a pipe containing a single circumferential crack when the uncertainty over the nominal crack profile is considered as an addition variation. As shown in Fig. 14(c) and (d), through the numerical discretization scheme implemented, the uncertainty associated with the maximum bending moment is proportional to the magnitude of the crack profile uncertainty and inversely proportional to the square root of the uncertainty sampling size (i.e, surface roughness vs. irregular crack profile) corresponding exactly to the mathematical formulation presented in equation (35). Obviously, similar results can be obtained in the case of a pipe containing multiple cracks. It is interesting to note that in the case of a pipe containing cracks with a constant depth crack profile, a one-to-one equivalence of the amplitude of the uncertainty between the additive case and the multiplicative case can be derived. Indeed, if we consider the normalized crack depth with respect to the pipe thickness (i.e. $a / t \in[0,1])$ for both the additive and multiplicative variation, we have,

$$
\frac{a_{j,+}(\xi)}{t}=\frac{a_{0, j}(\xi)}{t}+\epsilon_{j,+}(\xi),
$$

in the additive case with the additive profile variation $\epsilon_{j,+} \sim \mathcal{U}\left(-m_{\epsilon}, m_{\epsilon}\right)$. This additive variation can be expressed through a normalized uniform distribution $u_{+} \sim \mathcal{U}(0,1)$ such that $a_{j,+} / t$ reduces to

$$
\frac{a_{j,+}(\xi)}{t}=\frac{a_{0, j}(\xi)}{t}-m_{\epsilon}+2 m_{\epsilon} u_{+} .
$$

Similarly in the case of the multiplicative variation, the normalized crack depth with respect to the pipe thickness can be expressed as,

$$
\frac{a_{j, \times}(\xi)}{t}=\frac{a_{0, j}(\xi)}{t} \epsilon_{j, \times}(\xi)
$$

with the multiplicative profile variation $\epsilon_{j,+} \sim \mathcal{U}\left(1-r_{\epsilon}, 1+r_{\epsilon}\right)$. Again, this multiplicative variation can be expressed through a normalized uniform distribution $u_{\times} \sim \mathcal{U}(0,1)$ such 
that $a_{j, \times} / t$ reduces to

$$
\frac{a_{j, \times}(\xi)}{t}=\left(1-r_{\epsilon}+2 r_{\epsilon} u_{\times}\right) \frac{a_{0, j}(\xi)}{t}
$$

The equivalence between the multiplicative and additive case is satisfied when

$$
\frac{a_{j,+}(\xi)}{t}=\frac{a_{j, \times}(\xi)}{t}
$$

when $u_{+}=u_{\times}$. The above logic simplifies in the case of a constant crack depth profile (i.e. $a_{0, j}(\xi)=a_{0, j}$ such that the equivalence between the additive variation case and the multiplicative case is obtained through the relation,

$$
m_{\epsilon} r_{\epsilon}=\frac{a_{0, j}}{t}
$$

Table 1 list the equivalence of uncertainty when it is treated as an additive variation and a multiplicative variation over the nominal crack profile for selected amplitudes with a constant crack depth profile of $a / t=0.7$. Fig. 15 shows the perfect match between both cases. Looking at the condition expressed in equation (41), we should note that this equivalence is valid through the whole crack only in the case of a constant crack depth. For other crack profiles, the ratio $a(\xi) / t$ changes with the depth and therefore changes the equivalence in equation (42) along the crack. As expected, as shown in Fig. 16, if we look at the relative error between the uncertainty treated as an additive variation and a multiplicative variation for the effect of the amplitude of the crack profile on the net section collapse moment in the case where the nominal crack profile is assumed constant for various uncertainty amplitudes (see Fig. 16(a)), and in the case where the nominal crack profile is assumed semi-elliptical for various uncertainty amplitudes (see Fig. 16(b)), we clearly see that in the case of a constant crack depth, the relative is exactly null, while in the case of semielliptical crack profiles, differences exist depending on how the uncertainty is treated.

This exercise shows that, regardless of the way uncertainty on the crack profile is dealt with, the uncertainty associated with the surface roughness of the crack profile has little to 
no effect on the maximum bending moment, while the uncertainty associated with irregular shape profiles has a large effect on the estimation of the maximum bending moment. The idealized case of nominal crack profiles may not necessarily be the most conservation estimation of the failure bending moment.

\subsection{General remarks}

One of the problems with the application of limit load solutions for circumferential surface cracked pipes, even in the case of very ductile materials such as stainless steel, is that these solutions tend to be non-conservative for short angle cracks or when other failure mechanisms (e.g., ductile fracture or necking) control failure. The reason for the non-conservatism for short angle cracks lies in the fact that the net section for such cracks cannot achieve full plasticity prior to a crack breaking through the wall and becoming a through-wall crack. Furthermore, in the case of the idealized crack shapes, the closest representation of short deep cracks (i.e. cracks with a small $\theta$ and large $a / t$ ) considered in our analysis would be circular shaped cracks. For that short range of short angles, an elastic-plastic fracture mechanics method is necessary for these small cracks. For example, Kurihara et al. (1988) observed this phenomenon while performing tests on a short surface cracked pipe subjected to bending. Kurihara et al. (1988) proposed a semi-empirical correction to the limit-load criterion on the basis of test results. This limit-load criterion gives a conservative prediction by choosing an optimum parameter to empirically fit the experimental data for short and deep circumferential surface cracks. Miller (1988) notes in his review the distinction between global collapse and local collapse and refers to earlier work on instability of ligaments ahead of deep cracks in plates. Such an approach could be adapted in the present formulation and considered for short deep cracks. Alternatively, similar to the approach used in codes such as API 579 (Anderson and Osage, 2000) or BS 7910 (British Standards Institution, 2000), the present formulation could be augmented by 
including ductile fracture assessment explicitly in addition to collapse.

\section{Conclusion}

This manuscript presents a generalized net-section-collapse (NSC) failure criterion of circumferentially cracked pipes with multiple arbitrary-shaped cracks originally developed by Rahman and Wilkowski (1998) and extended to multiple crack formalism. The methodology uses the equilibrium of axial forces and the moment equilibrium for the cracked pipe to derive the limit-load analysis of cracks with any arbitrary shape. Closed form solutions are provided for the maximum moments of cracked pipes containing multiple cracks with idealized shapes, namely constant depth, semi-elliptical and parabolic crack profiles. In the case of arbitrarily-shaped cracks, it is necessary to use a discretization scheme for the profiles of the cracks in order to come up with numerical estimates of the limit-loads.

Numerical calculations on various crack shapes and configurations were performed. The results showed that significant differences exist in limit-load predictions for pipes when crack profiles are approximated by constant-depth, semi-elliptical, or parabolic crack profiles.

In fact through a statistical analysis, it is demonstrated that, while the uncertainty associated with the surface roughness of the crack profile has little to no effect on the maximum bending moment, irregular crack shape profiles have a large effect on the estimation of the maximum bending moment. Quantification of the uncertainty on the limit-load is directly proportional to the magnitude of the crack profile uncertainty and inversely proportional to the square root of the uncertainty sampling size (i.e surface roughness vs. irregular crack profile), regardless of whether the uncertainty on the crack profile is assumed to be additive or multiplicative.

Finally, while the generalized NSC analysis presented in this manuscript shows good agreement with experimental data, it should be noted that the present method may not be 
well-suited for short surface cracks (especially short deep cracks), because the assumption that the cross section becomes fully plastic cannot be realized before the crack breaks through the wall even in fully ductile, tough materials such as stainless steel.

\section{Acknowledgments}

The authors would like to thank Dr. F.W. Brust, Dr. D.L. Rudland and Dr. K. Hasegawa for their valuable comments and suggestions during this work. Sandia is a multiprogram laboratory operated by Sandia Corporation, a Lockheed Martin Company, for the United States Department of Energy, under Contract No. DE-AC04-94AL85000. 


\section{References}

Anderson, T. L., Osage, D. A., 2000. API 579: a comprehensive fitness-for-service guide. International Journal of Pressure Vessels and Piping 77 (14), 953-963.

ASME Boiler and Pressure Vessel Code Section XI, 1997. Rules for inservice inspection of nuclear power plant components.

British Standards Institution, 2000. Bs 7910:1999: (incorporating amendment no.1) guide on methods for assessing the acceptability of flaws in metallic structures.

Cramlington, N. L., 1996. Life after inspection. In: Proceedings of the International Pipeline Conference. Vol. 1. American Society of Mechanical Engineers, p. 417.

Han, L.-H., He, S.-Y., Wang, Y.-P., Liu, C.-D., 1999. Limit moment of local wall thinning in pipe under bending. International Journal of Pressure Vessels and Piping 76 (8), 539 -542 .

Hasegawa, K., Saito, K., Iwamatsu, F., Miyazaki, K., 2007. Prediction of fully plastic failure stresses for pipes with multiple circumferential flaws. In: Proceedings of the ASME Pressure Vessels and Piping Conference. Vol. 1. pp. 415-419.

Japan Society of Mechanical Engineers, 2008. Code for nuclear power generation facilities: Rules on fitness-for-service for nuclear power plants JSME S NA1-2008.

Kanninen, M. F., Broek, D., Marschall, C. W., Rybicki, E. F., Sampath, S. G., Simonen, F. A., Wilkowski, G. M., 1976. Mechanical fracture predictions for sensitized stainless steel piping with circumferential cracks.[BWR]. Tech. rep., Battelle Columbus Labs., $\mathrm{OH}$ (USA). 
Kim, Y.-J., Oh, C.-K., Park, C.-Y., Hasegawa, K., 2006. Net-section limit load approach for failure strength estimates of pipes with local wall thinning. International Journal of Pressure Vessels and Piping 83 (7), 546-555.

Kim, Y.-J., Shim, D.-J., Huh, N.-S., Kim, Y.-J., 2002. Plastic limit pressures for cracked pipes using finite element limit analyses. International Journal of Pressure Vessels and Piping 79 (5), $321-330$.

Kim, Y.-J., Shim, D.-J., Nikbin, K., Kim, Y.-J., Hwang, S.-S., Kim, J.-S., 2003. Finite element based plastic limit loads for cylinders with part-through surface cracks under combined loading. International Journal of Pressure Vessels and Piping 80 (78), 527540.

Kurihara, R., Ueda, S., Sturm, D., 1988. Estimation of the ductile unstable fracture of pipe with a circumferential surface crack subjected to bending. Nuclear Engineering and Design 106 (2), 265-273.

Lei, Y., Budden, P. J., 2004. Limit load solutions for thin-walled cylinders with circumferential cracks under combined internal pressure, axial tension and bending. The Journal of Strain Analysis for Engineering Design 39 (6), 673-683.

Li, Y., Hasegawa, K., Onizawa, K., Cofie, N. G., 2010. Prediction of collapse stress for pipes with arbitrary multiple circumferential surface flaws. Journal of Pressure Vessel Technology 132 (6), 061204.

Miller, A. G., 1988. Review of limit loads of structures containing defects. International Journal of Pressure Vessels and Piping 32 (1), 197-327.

Office of Pipeline Safety, US Department of Transportation, 2004. Pipeline statistics. 
Oh, C.-K., Kim, Y.-J., Kim, J.-S., Jin, T.-E., 2008. Yield locus for circumferential partthrough surface cracked pipes under combined pressure and bending. Engineering Fracture Mechanics 75 (8), 2175-2190.

Rahman, S., 1997. Probabilistic fracture analysis of cracked pipes with circumferential flaws. International Journal of Pressure Vessels and Piping 70 (3), 223-236.

Rahman, S., 1998. Net-section-collapse analysis of circumferentially cracked cylinderspart II: idealized cracks and closed-form solutions. Engineering Fracture Mechanics $61(2), 213-230$.

Rahman, S., Wilkowski, G., 1998. Net-section-collapse analysis of circumferentially cracked cylinders-part I: arbitrary-shaped cracks and generalized equations. Engineering Fracture Mechanics 61 (2), 191-211.

RSE-M Code, 1997. Rules for in-service inspection of nuclear power plant components. Paris: AFCEN.

Staat, M., Vu, D. K., 2006. Limit loads of circumferentially flawed pipes and cylindrical vessels under internal pressure. International Journal of Pressure Vessels and Piping 83 (3), 188-196. 
Table 1: Equivalence of uncertainty when the it is treated as an additive variation and a multiplicative variation over the nominal crack profile for a constant crack depth profile

\begin{tabular}{|c|c|}
\hline \hline$\epsilon_{j,+}(\%)$ & Equivalent $\epsilon_{j, \times}(\%)$ \\
\hline $1 \%$ & $1 \% / 0.7 \simeq 1.43 \%$ \\
$5 \%$ & $5 \% / 0.7 \simeq 7.14 \%$ \\
$10 \%$ & $10 \% / 0.7 \simeq 14.29 \%$ \\
$20 \%$ & $20 \% / 0.7 \simeq 28.51 \%$ \\
$30 \%$ & $30 \% / 0.7 \simeq 42.86 \%$ \\
\hline \hline
\end{tabular}




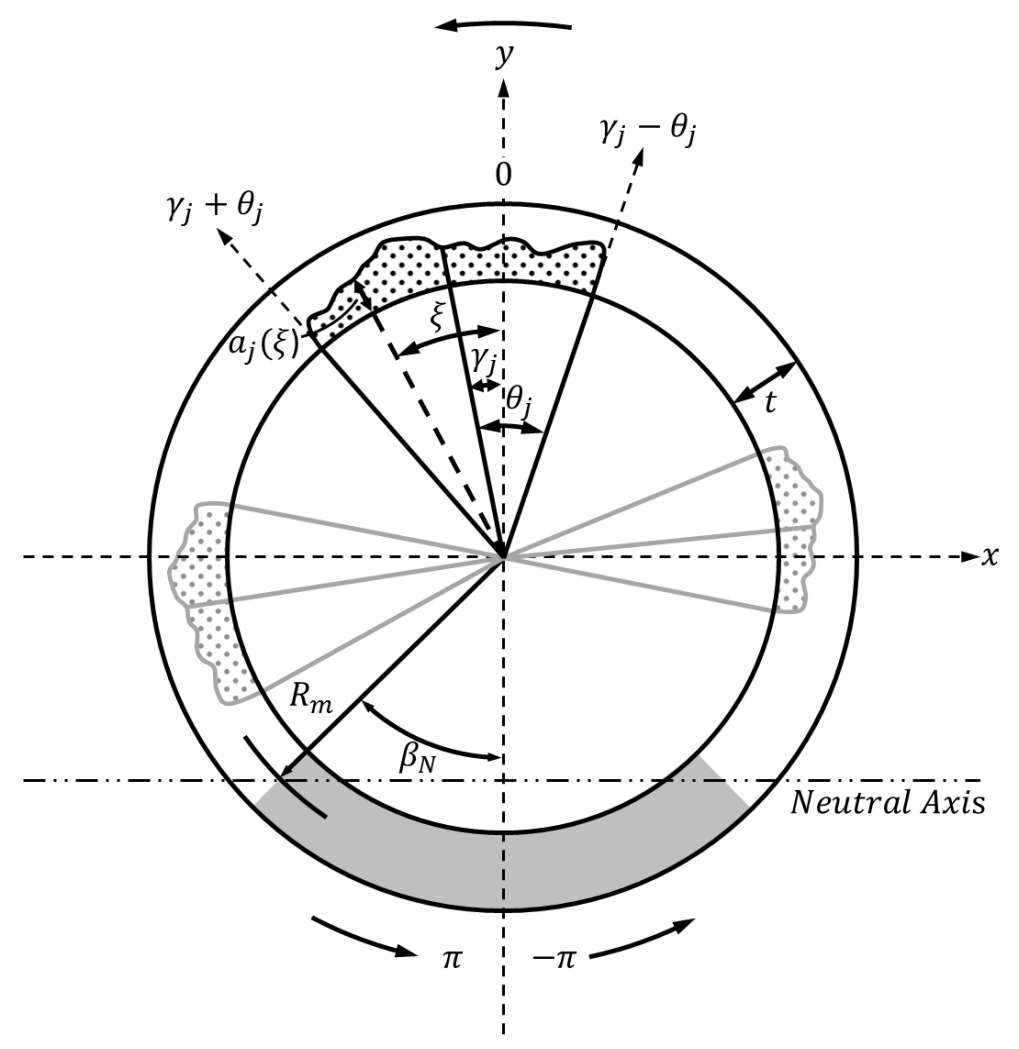

Figure 1: Archetype of arbitrary shaped cracks distributed around the circumference of a pipe. The greyshaded area of the pipe corresponds to the angular location of the neutral axis. 


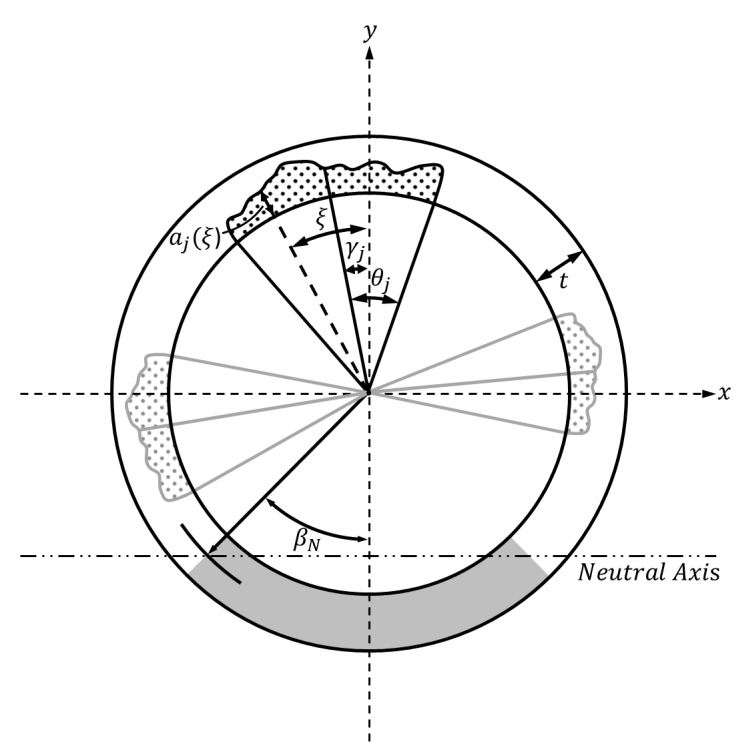

(a)
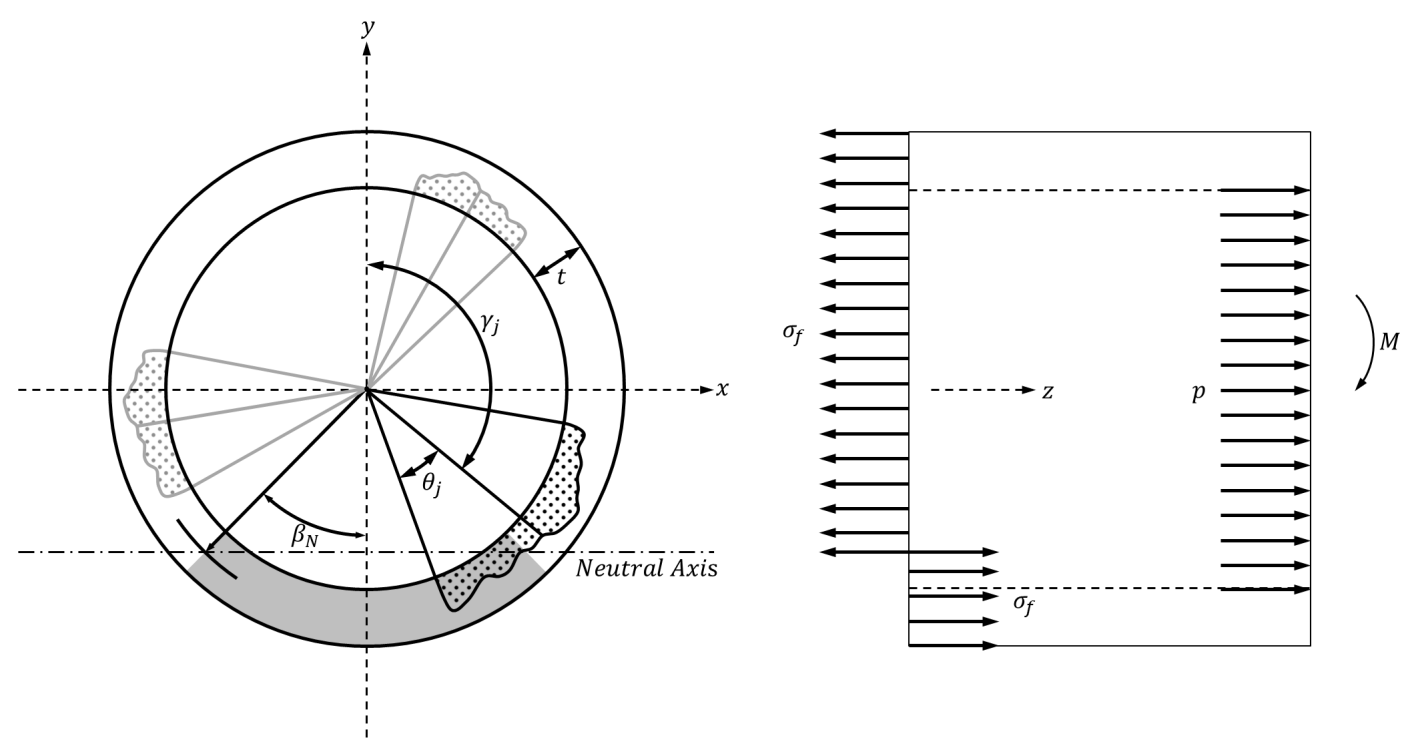

(b)

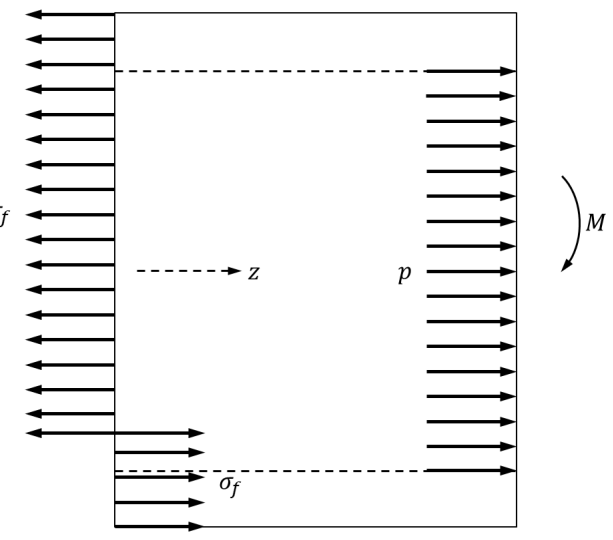

Figure 2: (a) All the cracks are in tension, (b) Some cracks are in compression. 


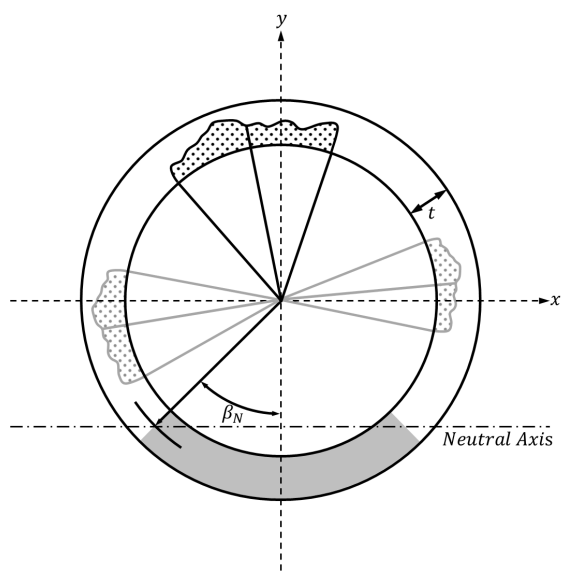

(a)

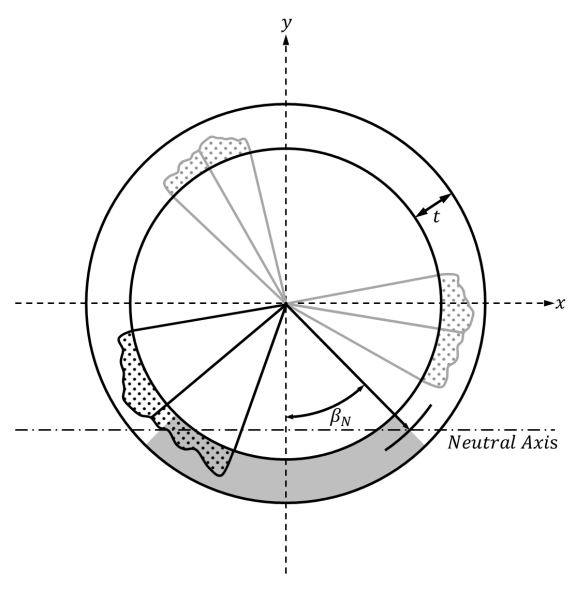

(c)

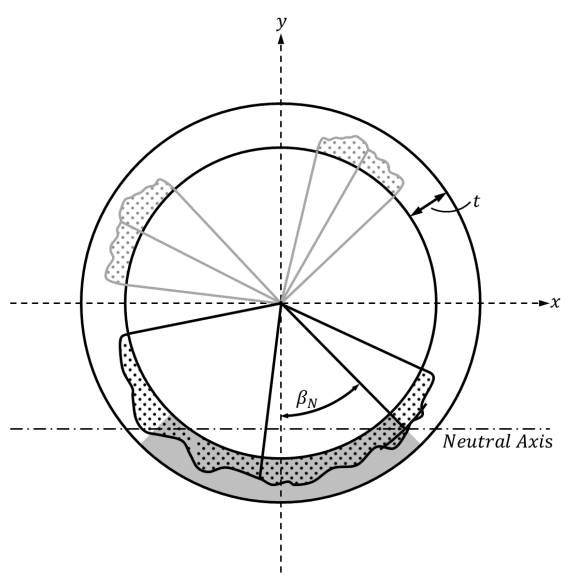

(e)

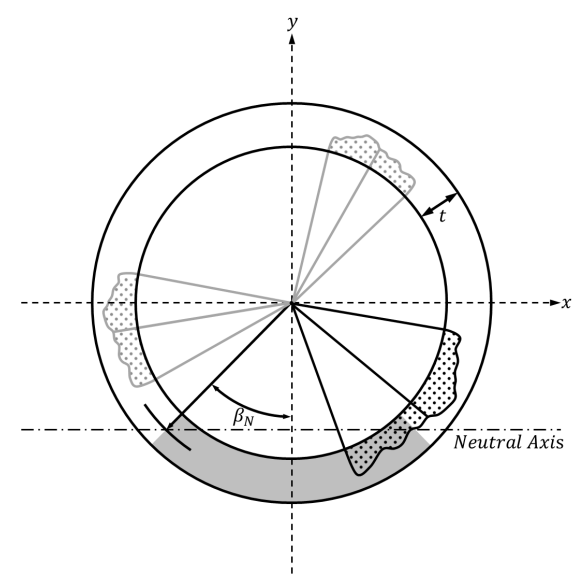

(b)

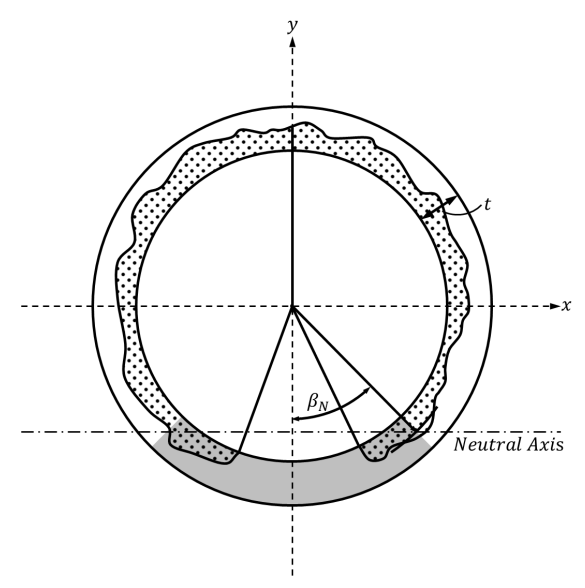

(d)

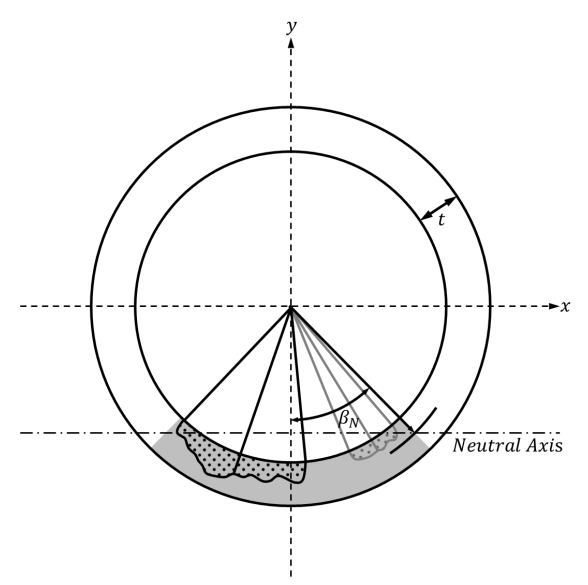

(f)

Figure 3: (a) Case 1: all the cracks are in tension, (b) Case 2: cracks on the left of the centerline are partially in compression, (c) Case 3: cracks on the right of the centerline are in partially in compression, (d) Case 4: cracks for which both crack tips straddle the compression zone, (e) Case 5: cracks for which both crack tips straddle the tensile zone, (f) Case 6: all cracks are in compression. 


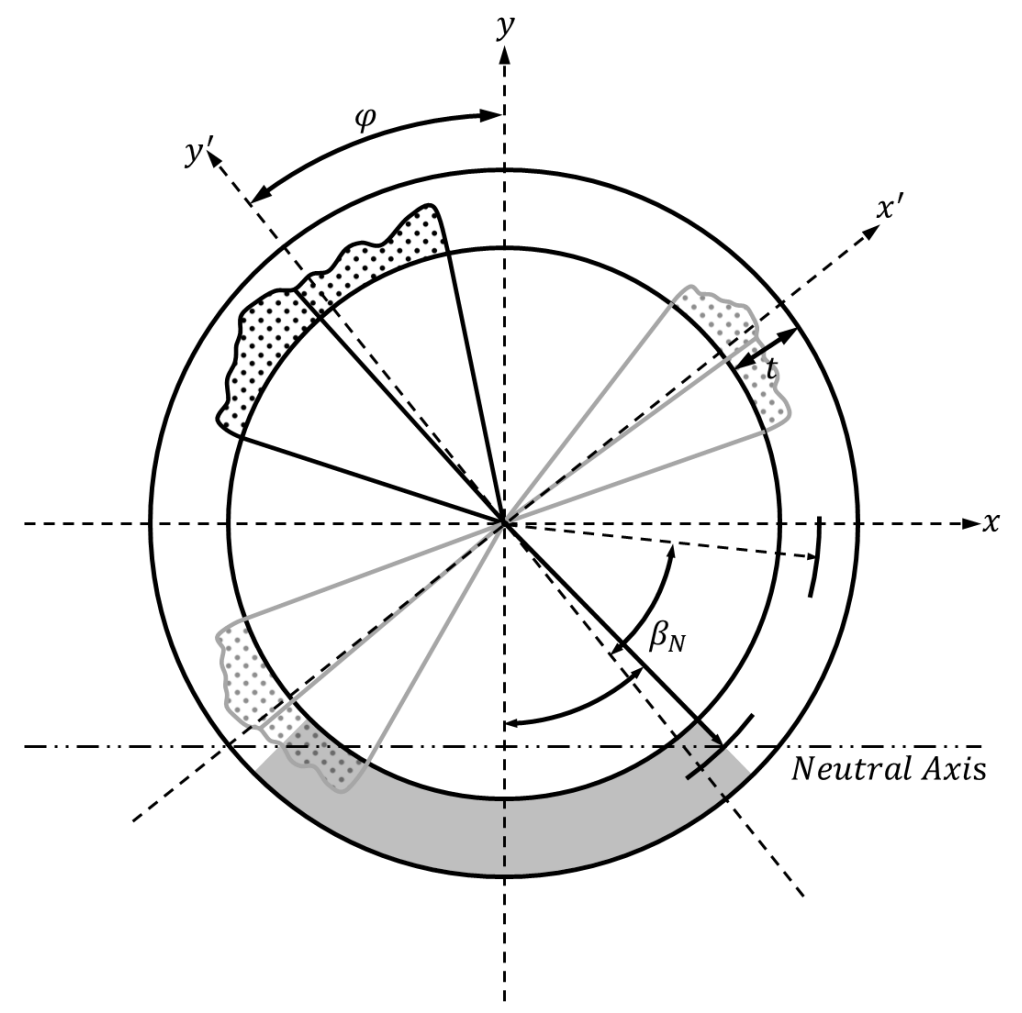

Figure 4: Convention for an asymmetrical configuration of cracks.

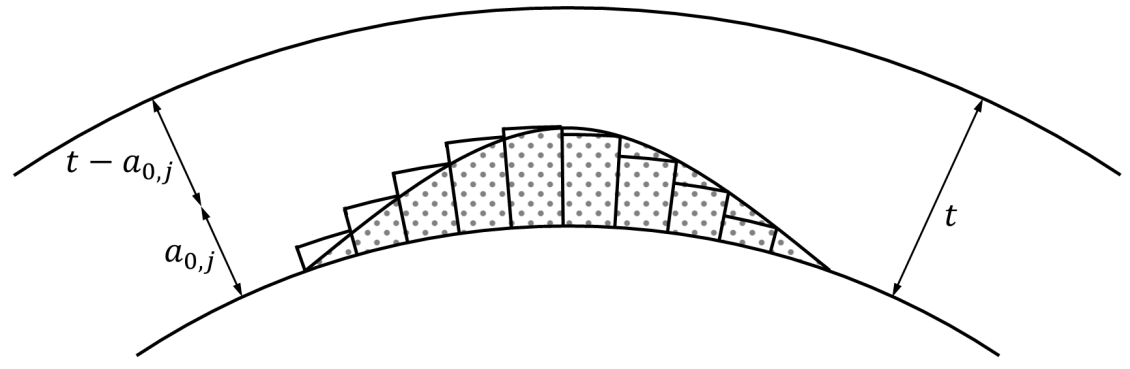

Figure 5: Illustration of the crack discretization. 


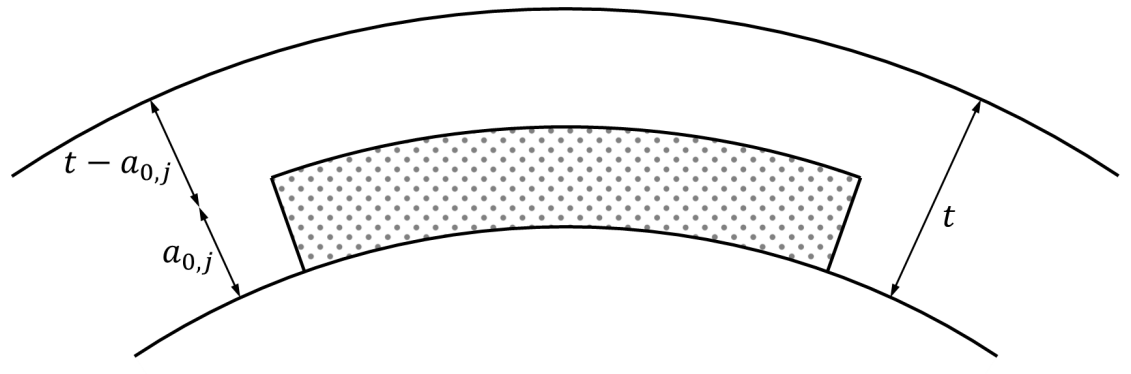

(a)

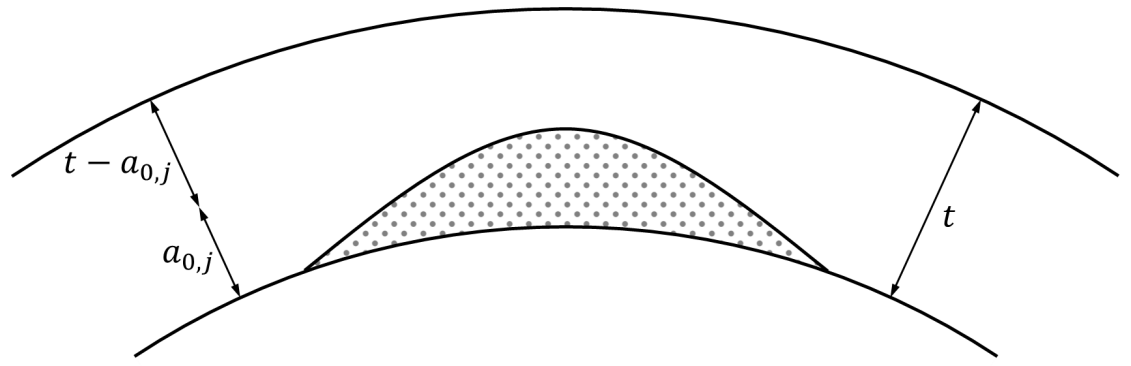

(b)

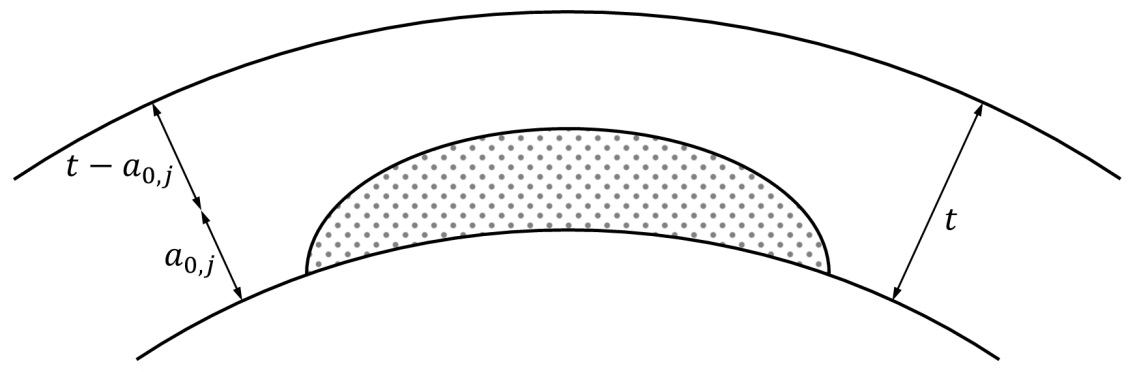

(c)

Figure 6: Idealized crack profiles typically used in pipe fracture analysis: (a) constant depth crack, (b) parabolic crack, (c) semi-elliptical crack. 


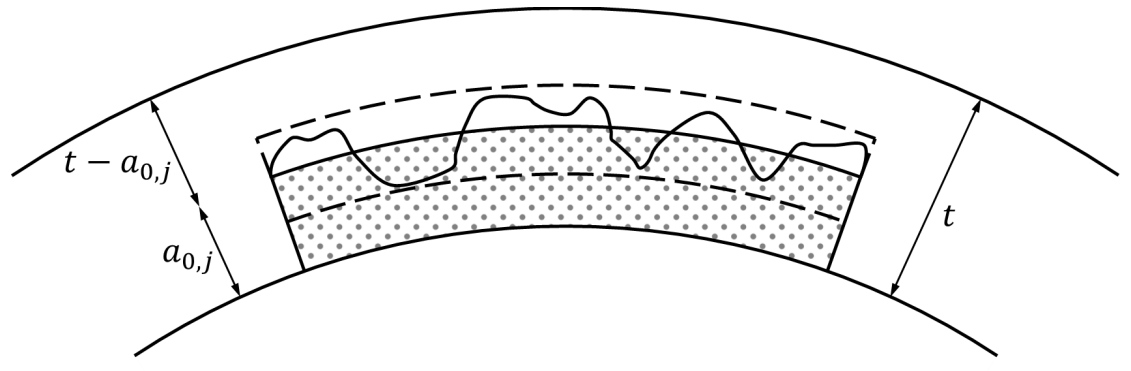

(a)

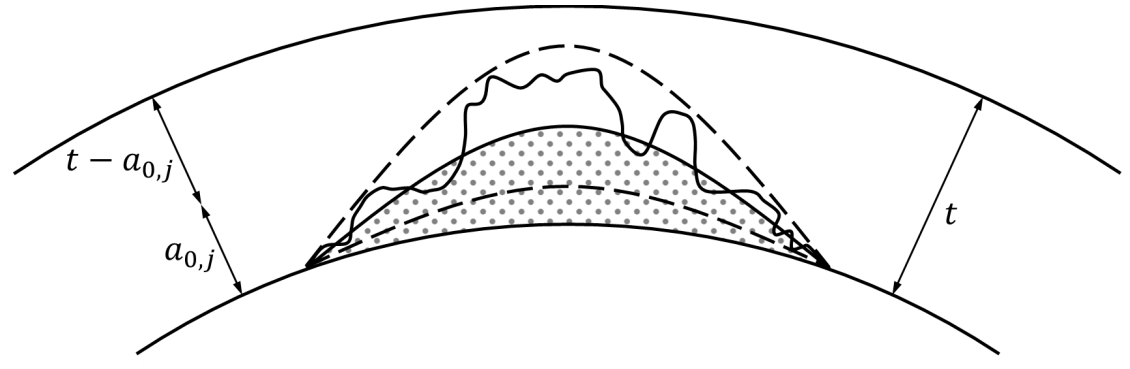

(b)

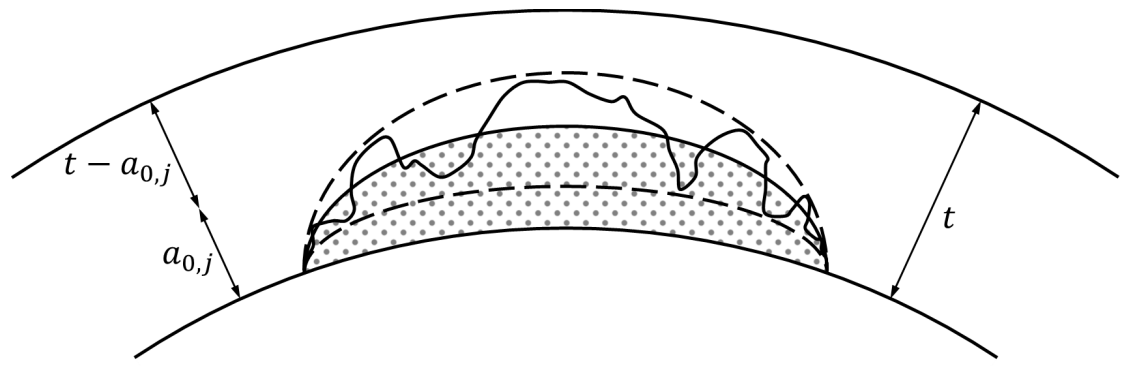

(c)

Figure 7: Illustration of the crack profile uncertainty deviating from the idealized cases: (a) constant depth crack, (b) parabolic crack, (c) semi-elliptical crack. 


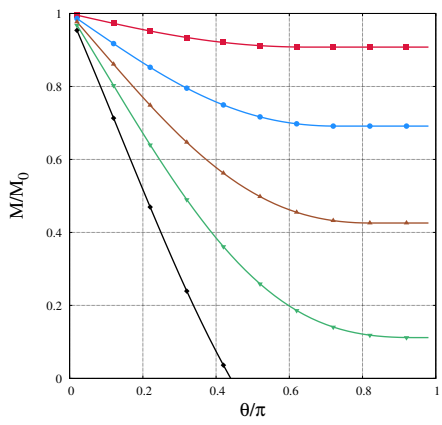

(a)

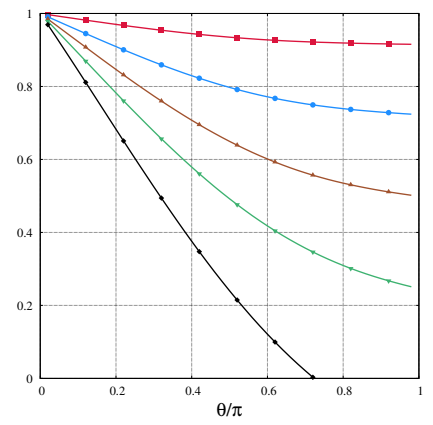

(b)

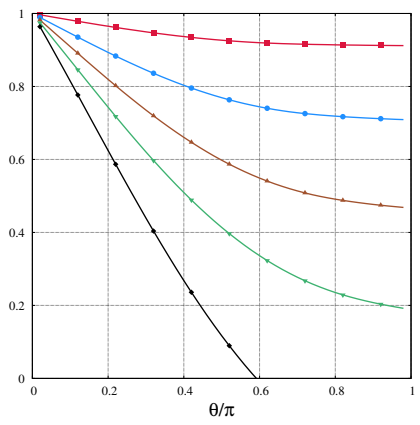

(c)

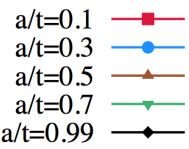

Figure 8: Variation of the normalized net section collapse moment with respect to crack size for a cracked pipe with a single crack for the idealized cases: (a) constant depth crack, (b) parabolic crack, (c) semielliptical crack. Parameters used are the following: $R_{m}=254 \mathrm{~mm}, t=25.4 \mathrm{~mm}, \sigma_{f}=300 \mathrm{MPa}$, and $p=$ 15.51 MPa. 


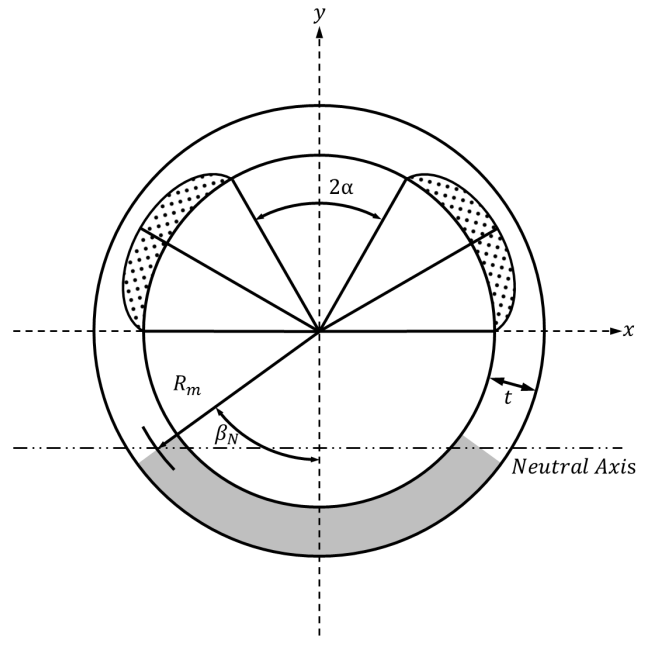

(a)

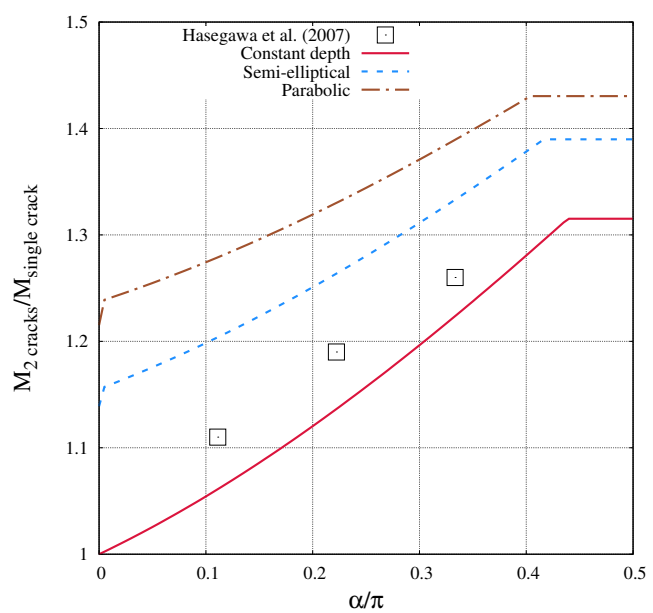

(b)

Figure 9: Comparison of the results from the generalized net section collapse analysis with experimental results from Hasegawa et al. (2007) using different idealized crack profiles (constant depth, parabolic and semi-elliptical crack profiles). Results are normalized with respect to the equivalent net section collapse moment for a single crack. Parameters used are the following: $R_{m}=53.1 \mathrm{~mm}, t=8.6 \mathrm{~mm}, \sigma_{f}=491 \mathrm{MPa}, p=$ $0 \mathrm{MPa}, N=2, a / t=0.74, \theta=\pi / 6$. 


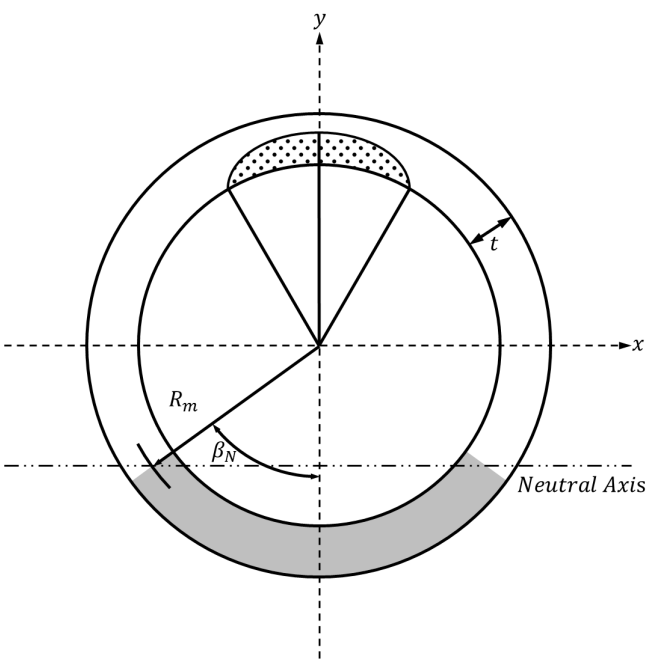

(a)

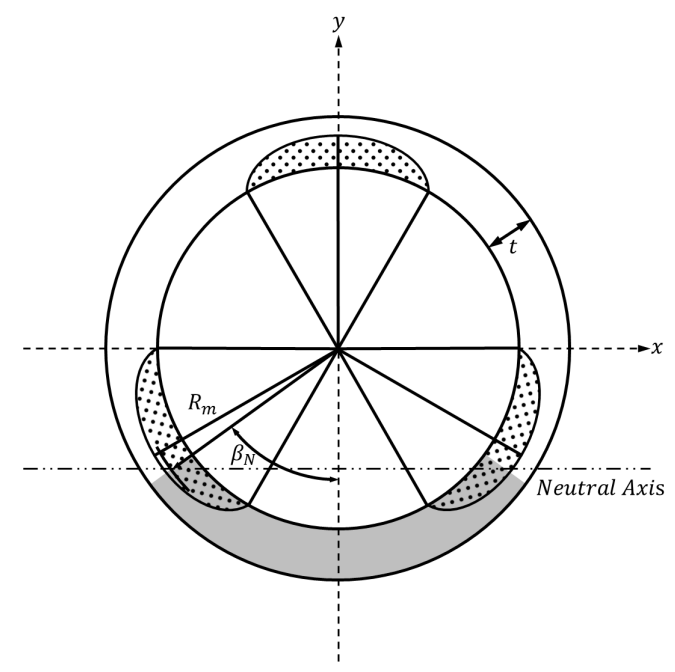

(c)

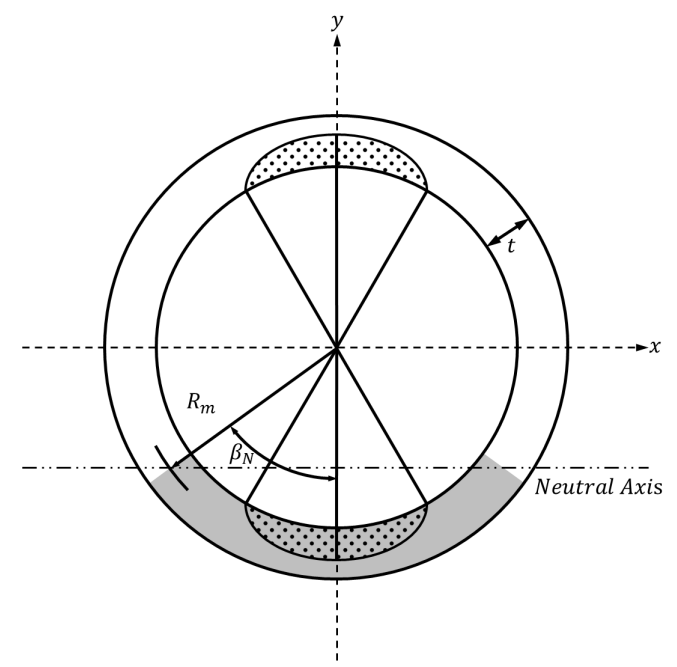

(b)

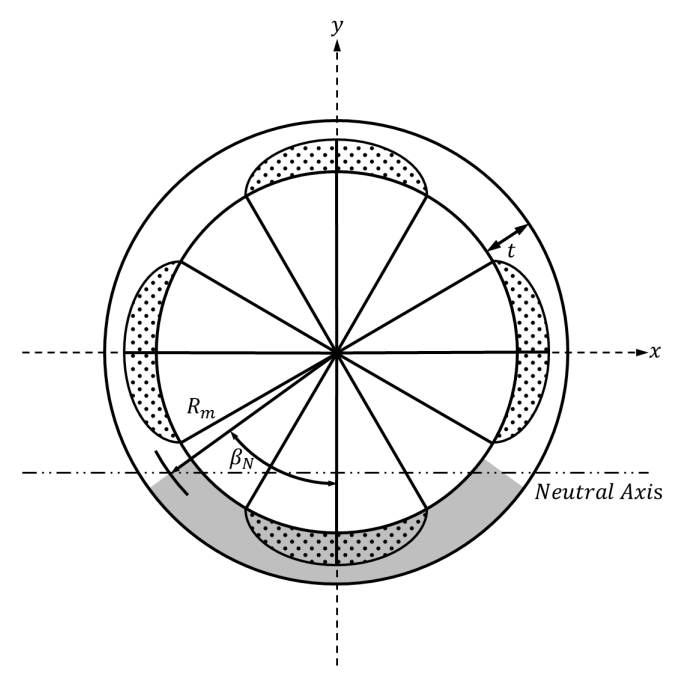

(d)

Figure 10: Crack configuration used in Fig. 11 and Fig. 12: (a) Single crack, (b) two cracks equally distributed around the circumference $(N=2)$, (c) three cracks equally distributed around the circumference $(N=3)$ and (d) four cracks equally distributed around the circumference $(N=4)$. 


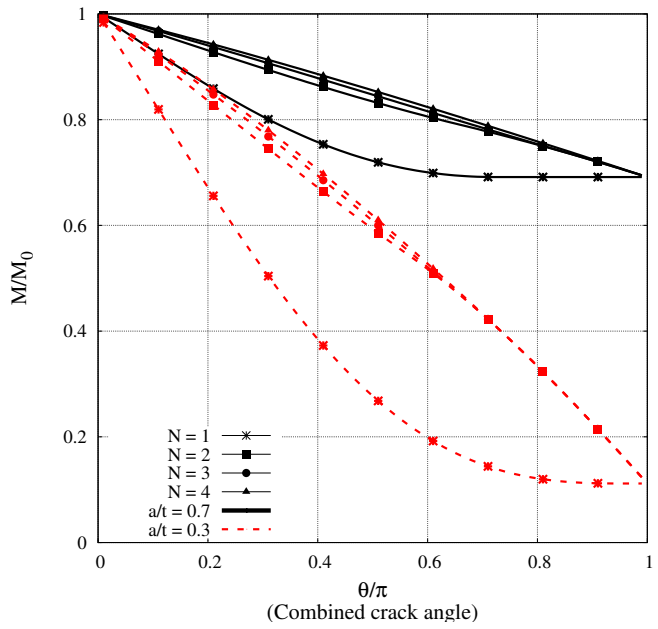

(a)

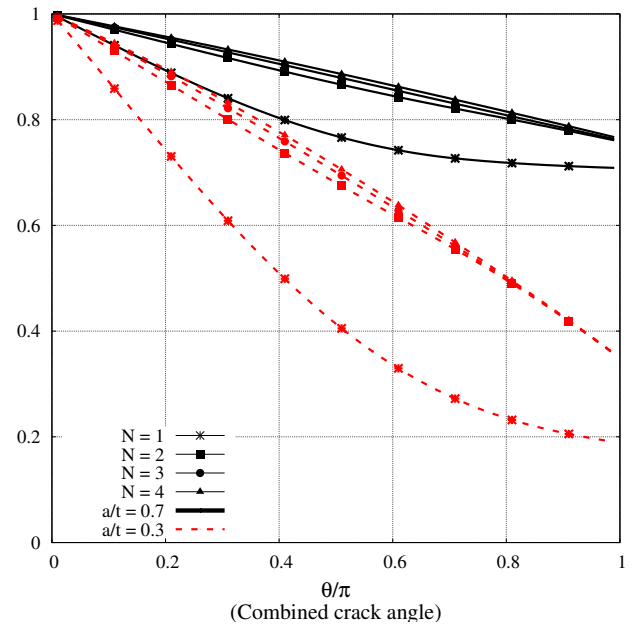

(b)

Figure 11: Effect of the number of cracks on the evolution of the net section collapse moment as a function of the combined circumferential crack coverage for (a) constant crack depth profile and (b) semi-elliptical crack profile. Solid black lines are for a ratio of $a / t=0.7$, dotted red lines for a ratio of $a / t=0.3$. Asterix symbol (*) denotes one crack $(N=1)$ distributed around the circumference, solid square symbol ( $(\mathbf{)})$ denotes two cracks $(N=2)$ distributed symmetrically around the circumference, solid circle symbol $(\bullet)$ denotes three cracks $(N=3)$ distributed symmetrically around the circumference, and solid triangle symbol $(\boldsymbol{\Lambda})$ denotes four cracks $(N=4)$ distributed symmetrically around the circumference. Crack configurations are presented in Fig. 10. Parameters used are same as the ones used in Fig. 8. 


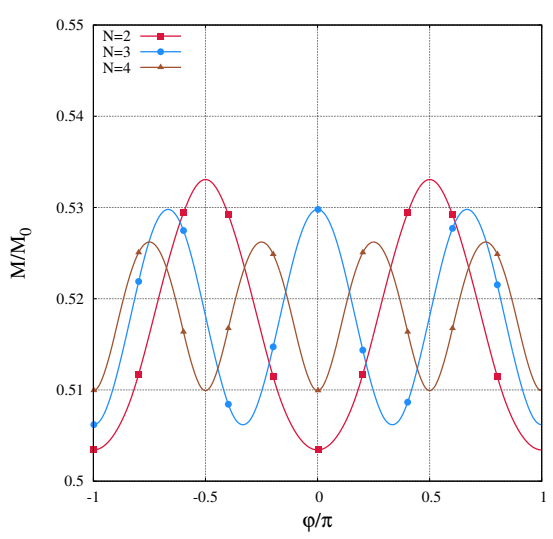

(a)

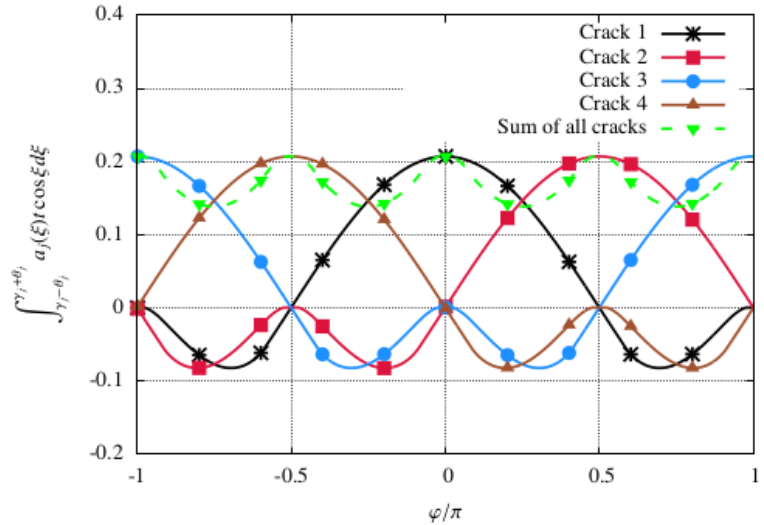

(b)

Figure 12: (a) Variation of the normalized net section collapse moment with respect to the rotation angle $\varphi$ for a pipe with multiple semi-elliptical cracks distributed around the circumference. (b) Contribution to the bending moment from each individual crack. Crack configurations are presented in Fig. 10. Parameters used are same as the ones used in Fig. 8. Additionally $a / t=0.7$ and $\theta / \pi=0.8$. 


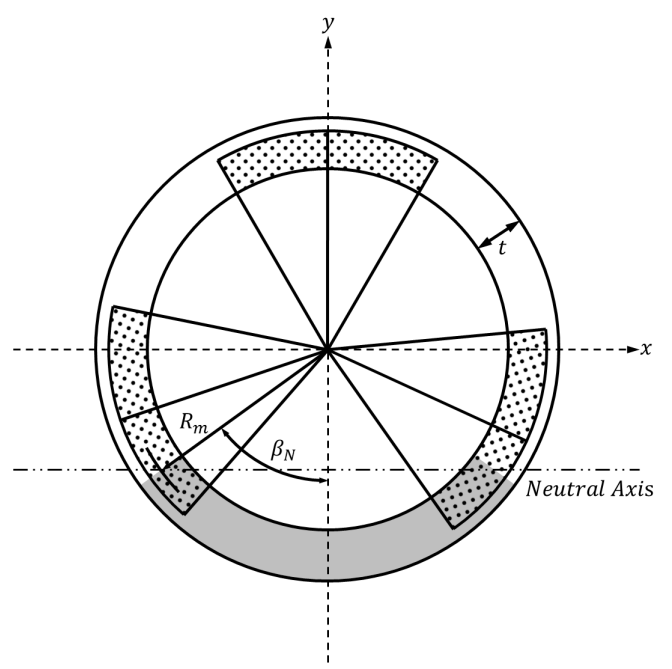

(a)

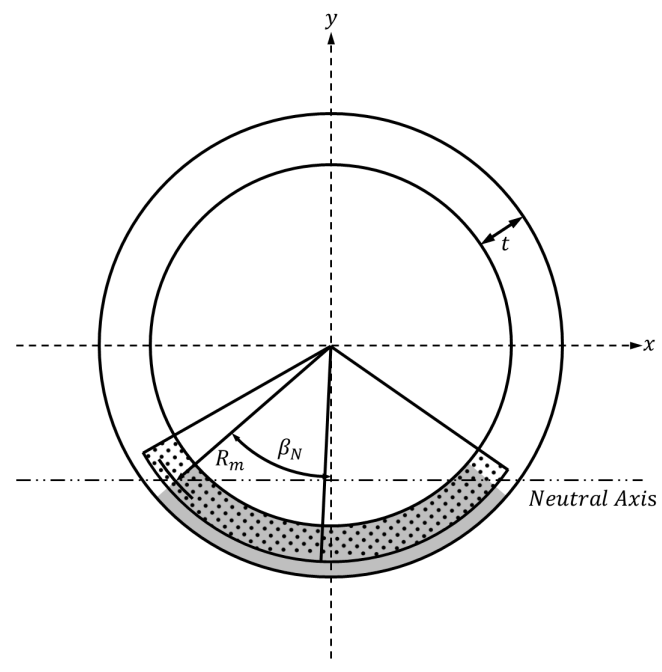

(c)

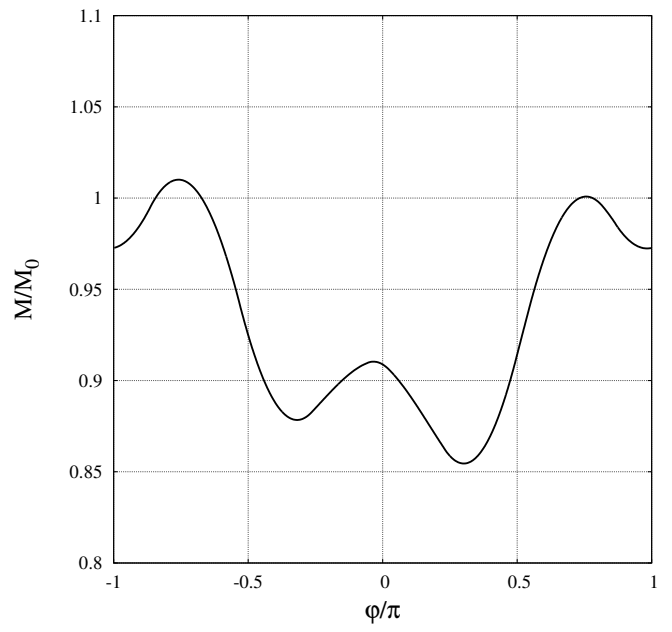

(b)

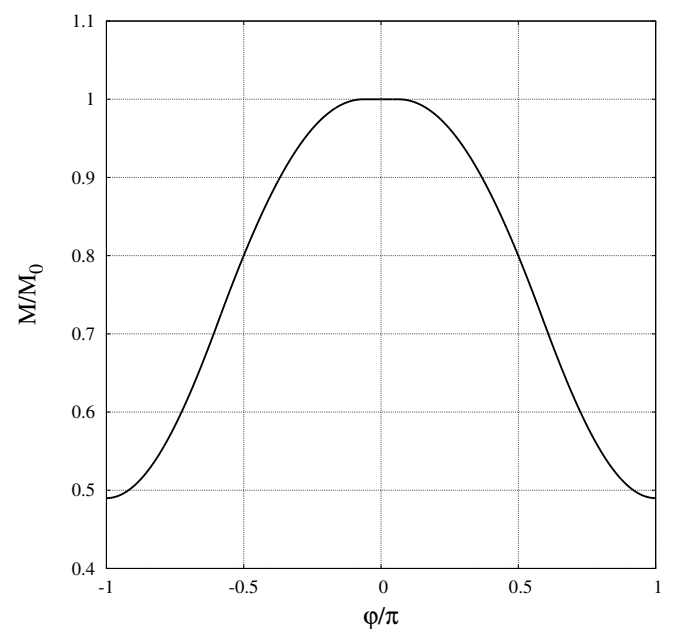

(d)

Figure 13: Illustration of the rotation angle $\varphi$ minimizing the bending moment for asymmetric distribution of cracks around the circumference of the pipe: (a) 3 cracks asymmetrically distributed around the circumference (cracks are positioned at $\gamma_{j}=0,1.9$, and -2.0 rad with a crack depth of $a_{0, j}=23.1 \mathrm{~mm}$ and crack angle $\theta_{j}=0.5 \mathrm{rad}, \forall j \in[1,3]$ ), (b) corresponding variation of the net section collapse bending moment as a function of the rotation angle $\varphi$, (c) Asymmetric configuration of a single crack straddling the neutral axis (cracks is positioned at $\gamma_{j}=3.1 \mathrm{rad}$ with a crack depth of $a_{0, j}=17.78 \mathrm{~mm}$ and crack angle $\theta_{j}=1.26 \mathrm{rad}$ ), (d) corresponding variation of the net section collapse bending moment as a function of the rotation angle $\varphi$. Parameters for both of these cases are $R_{m}=289.35 \mathrm{~mm}, t=30.9 \mathrm{~mm}, p=9.18 \mathrm{MPa}$, and $\sigma_{f}=257 \mathrm{MPa}$. 


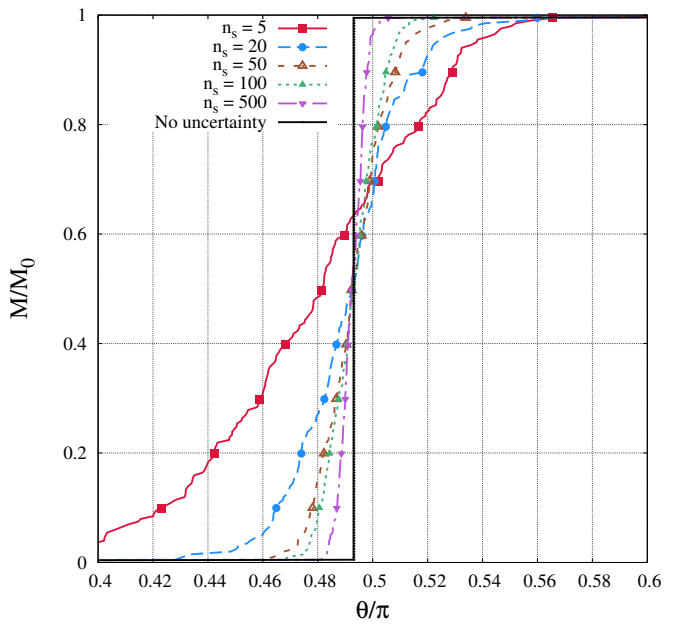

(a)

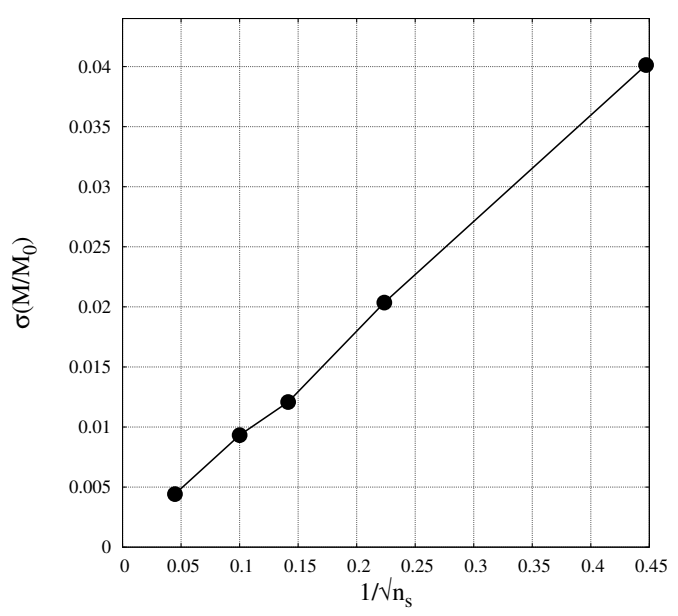

(c)

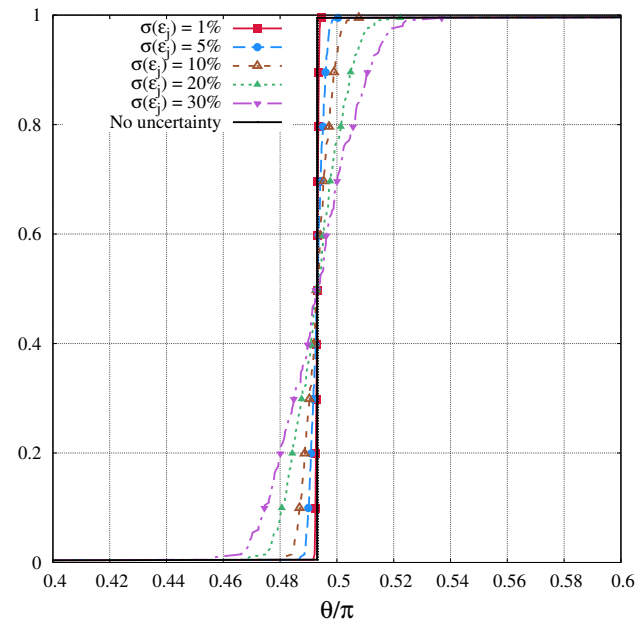

(b)

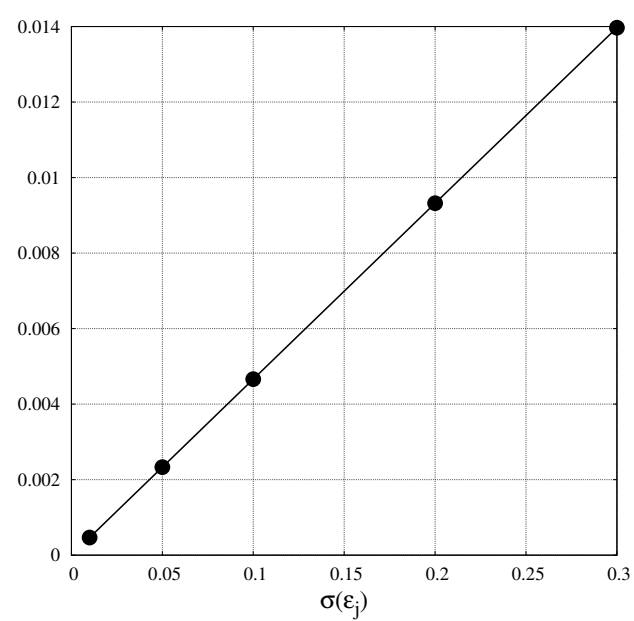

(d)

Figure 14: Effect of the uncertainty associated with (a) the sampling size $n_{s}$ (i.e period), and (b) the standard deviation $\sigma\left(\epsilon_{j}\right)$ (i.e. amplitude) in the case of a pipe containing a single circumferential crack. Linear correlations is observed between (c) the inverse square root of the sampling size on the uncertainty and the standard deviation of the NSC moment and (d) the uncertainty amplitude and the standard deviation of the NSC. Parameters used are same as the ones used in Fig. 8. Additionally $a / t=0.7$. 


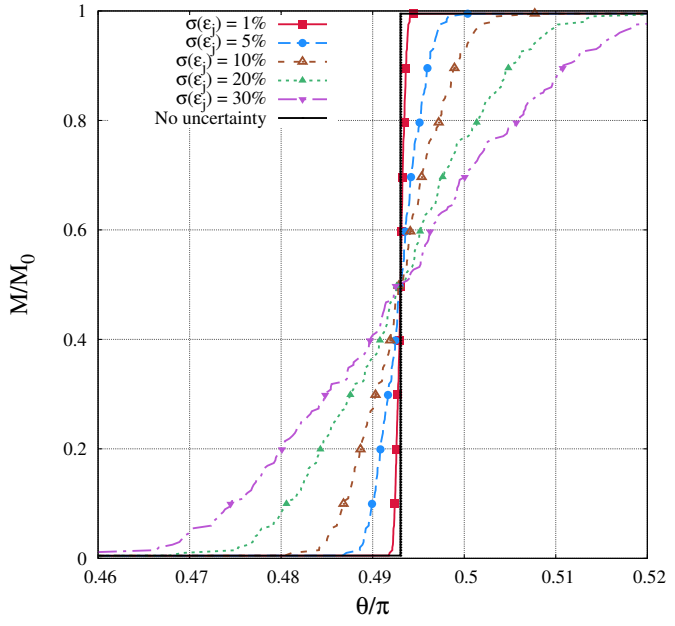

(a)

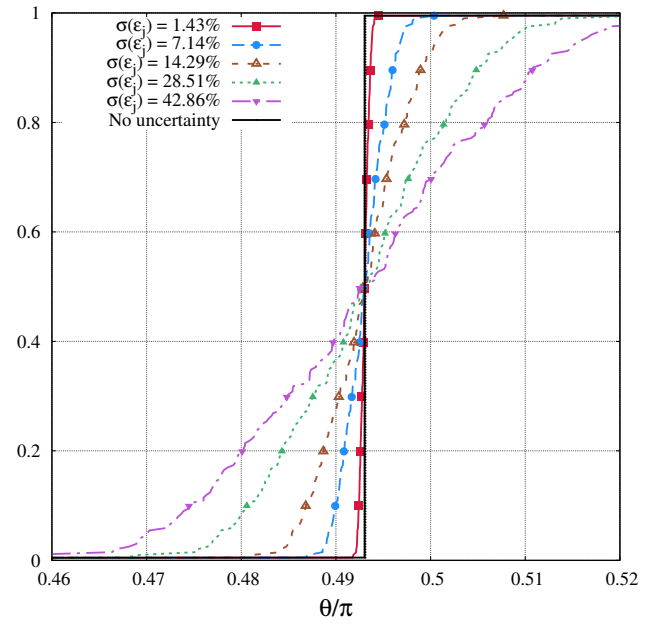

(b)

Figure 15: Equivalence for the effect of the amplitude of the crack profile on the net section collapse moment between (a) the uncertainty being treated an additive variation over the nominal crack profile, and (b) the uncertainty being treated an multiplicative variation over the nominal crack profile. Crack profile used is a constant depth crack profile. Parameters used are same as the ones used in Fig. 8. Additionally $a / t=0.7$. 


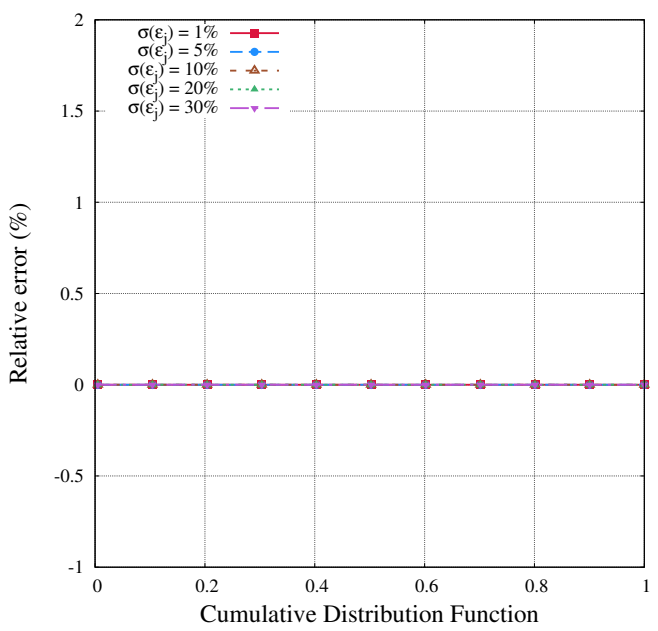

(a)

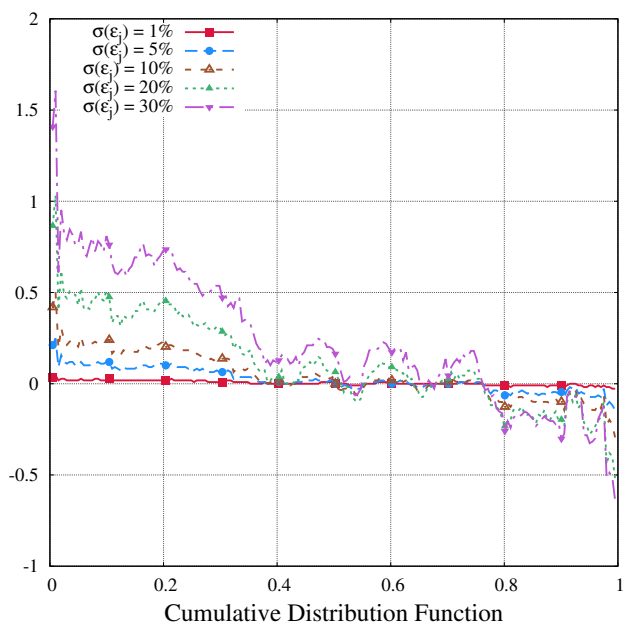

(b)

Figure 16: Relative error between the uncertainty treated as an additive variation and a multiplicative variation for the effect of the amplitude of the crack profile on the net section collapse moment: (a) in the case the nominal crack profile is assumed constant for various uncertainty amplitudes, and (b) in the case the nominal crack profile is assumed semi-elliptical for various uncertainty amplitudes. Parameters used are same as the ones used in Fig. 8. Additionally $a / t=0.7$. 